Pacific Journal of Mathematics

BOUNDED OPERATOR APPROACH TO TOMITA-TAKESAKI Marc ARISTide RIEFFel and Alfons VAN baEL 


\title{
A BOUNDED OPERATOR APPROACH TO TOMITA-TAKESAKI THEORY
}

\author{
Marc A. Rieffel and Alfons Van Daele
}

Let $M$ be a von Neumann algebra. The Tomita-Takesaki theory associates with each cyclic and separating vector for $M$ a strongly continuous one-parameter group, $\Delta^{i t}$, of unitary operators and a conjugate-linear isometric involution, $J$, of the underlying Hilbert space, such that $\Delta^{i t} M \Delta^{-i t}=M$ and $J M J=M^{\prime}$, where $M^{\prime}$ denotes the commutant of $M$.

The present paper has two purposes. In the first half of the paper we show that the operators $\Delta^{i t}$ and $J$ can, in fact, be associated with any fairly general real subspace of a complex Hilbert space, and that many of their properties, for example the characterization of $\Delta^{i t}$ in terms of the K.M.S. condition, can be derived in this less complicated setting. In the second half of the paper we show, by using some of the ideas from the first half, that a simplified proof of the Tomita-Takesaki theory given recently by the second author can be reformulated entirely in terms of bounded operators, thus further simplifying it by, among other things, eliminating all considerations involving domains of unbounded operators.

1. Introduction. Our approach is motivated by the following observation. Let $M$ be a von Neumann algebra on a Hilbert space $\mathscr{H}$, and let $\omega$ be a cyclic and separating vector for $M$. Let $M_{s}$ denote the collection of self-adjoint elements of $M$ and let $\mathscr{K}$ denote the closure of $M_{s} \omega$. Then $\mathscr{K}$ is a real subspace of $\mathscr{H}$ which can easily be shown to have the properties that $\mathscr{K} \cap i \mathscr{K}=\{0\}$ and $\mathscr{K}+i \mathscr{K}$ is dense in $\mathscr{H}$ (see Proposition 4.1). In [10] we found that the positions of $\mathscr{K}$ and $i \mathscr{K}$ were closely related to questions concerning an operator algebra and its commutant. In the present paper we find that the operators $\Delta^{i t}$ and $J$ depend only on the relative positions of $\mathscr{K}$ and $i \mathscr{K}$, and, in particular, can be defined in terms of the projections on these two subspaces. More generally, the operators $\Delta^{i t}$ and $J$ can be associated with any such real subspace, whether or not it comes from a von Neumann algebra. Because of this, it turns out that this subject is closely related to earlier work of Dixmier [4] and Halmos [7] on pairs of subspaces of Hilbert spaces. In fact, we digress at the end of the next section to show that our approach gives slightly simpler proofs of some of their main results.

In writing this paper we have addressed ourselves to those who are already familiar with the original approaches to Tomita-Takesaki 
theory $[13,14,16,17]$ as well as recent simplifications $[18,19,22]$. But those who wish to learn the subject (and are familiar with the early parts of [5]) will have no difficulty in reading this paper if they simply disregard statements which refer to these earlier approaches or to unbounded operators.

The content of the next sections is as follows. The second section treats pairs of subspaces of real Hilbert spaces, while the third treats one real subspace of a complex Hilbert space. In the fourth section we prove the main results of Tomita-Takesaki theory for the case of a von Neumann algebra with cyclic and separating vector, while in the fifth we treat left Hilbert algebras.

Most of the research for this paper was conducted while we were both visiting at the University of Pennsylvania. We would like to thank the members of the Department of Mathematics there for their warm hospitality during our visits. This research was partially supported by National Sciences Foundation grants GP-30798X and GP-28976.

2. Two subspaces. Since we will need to study real subspaces of a complex Hilbert space, we will frequently need to view a complex Hilbert space as a real Hilbert space, by taking the real part of its inner product. Thus, much of the information we need only concerns pairs of subspaces in real Hilbert spaces, and accordingly it is this subject that we study in this section.

Let $\mathscr{C}$ be a real Hilbert space, and let $\mathscr{K}$ and $\mathscr{L}$ be two (closed) subspaces of $\mathscr{C}$ which satisfy the nondegeneracy condition that $\mathscr{K} \cap \mathscr{L}=\{0\}$ and $\mathscr{K}+\mathscr{L}$ is dense in $\mathscr{H}$. We can define a (usually unbounded) operator, $S$, with (dense) domain $\mathscr{K}+\mathscr{L}$ by

$$
S(\xi+\eta)=\xi-\eta
$$

for $\xi \in \mathscr{K}$ and $\eta \in \mathscr{L}$. The operator $S$ is well-defined because $\mathscr{K} \cap$ $\mathscr{L}=\{0\}$. In fact it is a simple matter to check that $S$ is a closed operator because $\mathscr{K}$ and $\mathscr{L}$ are closed.

It is easily seen that if $\mathscr{K}$ is the closure of $M_{s} \omega$ as in the introduction, and if $\mathscr{L}=i \mathscr{K}$, then we obtain exactly the usual operator $S$ of Tomita-Takesaki theory (see the appendix).

Returning to the general setting, we could form the polar decomposition, $S=J \Delta^{1 / 2}$, of $S$, to obtain operators analogous to those of Tomita-Takesaki theory, and we could immediately study these operators further. But we prefer instead to show how to work in terms of bounded operators only, so that $S$ need not ever be defined.

Definition 2.1. Let $P$ and $Q$ denote the orthogonal projections onto $\mathscr{K}$ and $\mathscr{L}$ respectively. Let 


$$
R=P+Q,
$$

and let

$$
J T=P-Q,
$$

be the polar decomposition of $P-Q$, where $T$ is the positive operator and $J$ is the partial isometry.

We now collect together some elementary properties of $R, T$ and $J$ which will be used throughout this paper.

Proposition 2.2. With $R, T$ and $J$ defined as above, we have

1. Both $R$ and $2-R$ are injective, and $0 \leqq R \leqq 2$.

2. $T=R^{1 / 2}(2-R)^{1 / 2}$, and $T$ is injective.

3. $J$ is a self-adjoint orthogonal operator, so that $J^{2}=1$.

4. $T$ commutes with $P, Q, R$ and $J$.

5. $J P=(1-Q) J, J Q=(1-P) J$

$$
J R=(2-R) J .
$$

Proof. (1) It is clear that $0 \leqq R \leqq 2$. Suppose that $R \xi=0$. Then

$$
\|P \xi\|^{2}+\|Q \xi\|^{2}=\langle P \xi, \xi\rangle+\langle Q \xi, \xi\rangle=\langle R \xi, \xi\rangle=0,
$$

so that $P \xi=0=Q \xi$. Thus $\xi$ is orthogonal to both $\mathscr{K}$ and $\mathscr{L}$, and so $\xi=0$ since $\mathscr{K}+\mathscr{L}$ is dense. Hence $R$ is injective. Now the pair $\left(\mathscr{K}^{\perp}, \mathscr{L}^{\lrcorner}\right)$also satisfies the nondegeneracy condition, and the above argument can be applied to the projections $1-P$ and $1-Q$ to show that $2-R$ is injective.

(2) A simple calculation shows that

$$
T^{2}=P-P Q-Q P+Q=R(2-R),
$$

so that $T=R^{1 / 2}(2-R)^{1 / 2}$. Then $T$ is injective since both $R$ and $2-R$ are.

(3) Since $P-Q$ is self-adjoint, $J$ must be self-adjoint. Since $T$ is injective, $J$ must be injective. Thus $J^{2}=1$.

(4) Since $P-Q$ is self-adjoint, $T$ and $J$ commute. Now

$$
T^{2} P=P(P-Q)^{2} P=P(1-Q) P=P T^{2},
$$

so that $P$ commutes with $T^{2}$ and herce with $T$. Similarly $Q$ and $T$ commute. Thus $R$ and $T$ commute.

(5) $T J P=(P-Q) P=(1-Q)(P-Q)=(1-Q) T J=T(1-Q) J$. Since $T$ is injective, it follows that $J P=(1-Q) J$. The second relation follows by taking the adjoint of both sides of this relation. The third relation follows by adding the first two. 
We remark that property (5) shows that $J \mathscr{K}=\mathscr{L}^{\perp}$ and $J \mathscr{L}=$ $\mathscr{K}^{\perp}$, so that $J$ carries the pair $(\mathscr{K}, \mathscr{L})$ to the pair $\left(\mathscr{L}^{\perp}, \mathscr{K}^{\perp}\right)$. Under this transformation $R$ is replaced by $2-R$, while $T$ is left invariant since it commutes with $J$.

It is interesting to see how our operators $R, T$ and $J$ are related to the operators $S, F, \Delta$ and $J$ of the usual theory, but since we will not need to use these relations we have relegated a discussion of this matter to the appendix of this paper. Let us mention here only that the operators $J$ coincide, while $\Delta=(2-R) R^{-1}$.

Finally, we remark that all of these operators are closely related to the operators $A$ and $B$ on pages 390 and 391 of [4], while property 2 should be compared with Proposition 3 of [4]. Also, our operator $R$, or at least $R / 2$, does make a fleeting appearance in Takesaki's monograph [13] in the form of the operator $K$ defined on page 32 of [13].

We would now like to characterize the operator $J$. We will not need this characterization later, but it is interesting to see how such a characterization is obtained in our context. A characterization within the context of Tomita-Takesaki theory, with $J$ defined in terms of the polar decomposition of $S$, has been given by Araki [1] and Woronwicz [21] (see also p. 254 of [20]), and their proofs, involving unbounded operators, can easily be adapted to the present context. But actually, the desired result already essentially appears as Theorem 1 of [4], with a proof involving only bounded operators. We give here a proof which is slightly simpler than Dixmier's, in that it involves only the canonically defined operators introduced above, and does not involve first choosing an arbitrary isometry of $\mathscr{K}$ onto $\mathscr{L}^{\perp}$ as he does.

Proposition 2.3. The operator $J$ defined above is the unique self-adjoint orthogonal operator with the following properties:

(1) $J \mathscr{K}=\mathscr{L}^{\perp}\left(\right.$ so $\left.J \mathscr{L}=\mathscr{K}^{\perp}\right)$.

(2) $\langle J \xi, \xi\rangle \geqq 0$ for all $\xi \in \mathscr{K}$, while $\langle J \eta, \eta\rangle \leqq 0$ for all $\eta \in \mathscr{L}$.

Proof. We already know from part 5 of Proposition 2.2 that $J$ satisfies the first property. Now from this

$$
P J P=P(1-Q) J=P(P-Q) J=P T .
$$

But $P$ and $T$ are positive operators which commute, and so $P T \geqq 0$. Thus $P J P \geqq 0$. A similar calculation shows that $Q P Q \leqq 0$. It follows that $J$ satisfies the second property also.

Suppose now that $K$ is another self-adjoint orthogonal operator on $\mathscr{H}$ for which properties 1 and 2 above hold, so that 


$$
K P=(1-Q) K, \quad P K P \geqq 0, \text { and } Q K Q \leqq 0 .
$$

We must show that $K=J$. Now

$$
(J K) P=J(1-Q) K=P(J K),
$$

so that $J K$ commutes with $P$. A similar calculation shows that $J K$ also commutes with $Q$. Thus $J K$ commutes also with $R, T$, and $J$. In particular, $K$ and $J$ commute with each other. Now

$$
\begin{aligned}
R T(K J) & =(P+Q)(P-Q) K \\
& =(P(1-Q)-Q(1-P)) K=P K P-Q K Q \geqq 0 .
\end{aligned}
$$

But $R T$ is injective and positive, while $K J$ is orthogonal, so by the uniqueness of polar decompositions $K J=1$, so $K=J$.

We remark that both in the application of this result to TomitaTakesaki theory and in the setting of the next section we will have $\mathscr{L}=i \mathscr{K}$. Thus the requirement that $Q K Q \leqq 0$ in property 2 above will follow from the requirement that $P K P \geqq 0$. Also, while the above proposition at first only yields $\operatorname{Re}\langle J \xi, \xi\rangle \geqq 0$ for $\xi \in \mathscr{K}$, we also know that $\langle J \xi, \xi\rangle$ is real since $J \mathscr{K}^{\perp}=i \mathscr{K}^{\perp}$, so that again $\langle J \xi, \xi\rangle \geqq 0$.

In concluding this section we digress to indicate how the above approach can be used to give a proof of perhaps the most useful version of the main theorem of Halmos' paper [7], namely his Theorem 3. Our proof is simpler than his proof in that again it involves only the canonically defined operators introduced above, and does not involve first choosing an arbitrary isometry from $\mathscr{K}$ to $\mathscr{L}^{\perp}$ and then later compensating for this choice. Also our proof makes clear that the theorem is true for the case of real as well as complex scalars. We will not need this theorem later in this paper, but recent applications of this theorem where it is essential to be able to work with real scalars can be found in $[6,9]$, and our familiarity with this theorem helped us to arrive at some of the points of view used in the present paper.

THEOREM 2.4 (Theorem 3 of [7]). Let $\mathscr{H}$ be a Hilbert space (real or complex), and let $\mathscr{K}$ and $\mathscr{L}$ be two (closed) subspaces of $\mathscr{H}$ which are in general position, that is, the intersection of any two of $\mathscr{K}, \mathscr{L}, \mathscr{K}^{\perp}, \mathscr{L}^{\perp}$ is $\{0\}$. Then there is a Hilbert space $\mathscr{M}$, an operator, $C$, on $\mathscr{M}$ such that $0 \leqq C \leqq 1$ and $C$ and $1-C$ are injective, and an orthogonal (or unitary) operator from $\mathscr{H}$ onto $\mathscr{M} \oplus \mathscr{M}$ which carries $\mathscr{K}$ to the graph of $C$ and $\mathscr{L}$ to the graph of $-C$. 
Proof. Since $\mathscr{C}$ and $\mathscr{L}$ are in general position, the nondegeneracy conditions used earlier are satisfied, so we can define the operators $P, Q, R, T$ and $J$ as before, satisfying the properties of Proposition 2.2. But we now have more information. Specifically, the pair $\left(\mathscr{K}^{\perp}, \mathscr{L}\right)$ also satisfies the nondegeneracy condition, and from parts 2 and 3 of Proposition 2.2 applied to this pair it follows that $(1-P)-$ $Q=1-R$ is injective.

Let $\mathscr{C l}$ be the range of the spectral projection for $R$ corresponding to the interval $[1,2]$. From the fact that $J R J=2-R$, it is easily seen that $J \mathscr{C}$ is the range of the spectral projection of $R$ corresponding to the interval $[0,1]$. Moreover, since $0 \leqq R \leqq 2$ and since, as just shown above, 1 is not an eigenvalue for $R$, we must have that $J \mathscr{C}=\mathscr{C}^{\perp}$.

From the definition of $\mathscr{C l}$ we see that the restriction of $(2-R)^{1 / 2} R^{-1 / 2}\left(=\Delta^{1 / 2}\right)$ to $\mathscr{C l}$ is a bounded operator on $\mathscr{l}$, which we will denote by $C$. In fact it is clear that $0 \leqq C \leqq 1$ and that $C$ and $1-C$ are injective.

We claim that

$$
\mathscr{K}=(1+J C) \mathscr{L}, \mathscr{L}=(1-J C) \mathscr{K} .
$$

It is sufficient to prove the first of these equalities, since interchanging $\mathscr{K}$ and $\mathscr{L}$, and hence $P$ and $Q$, changes $J$ to $-J$ but has no effect on $R$ and so on $C$ or $\mathscr{K}$. Consider first $\xi \in \mathscr{l l}$. Then

$$
\begin{aligned}
2 P \xi=(R+J T) \xi & =\left(R+J(2-R)^{1 / 2} R^{1 / 2}\right) \xi \\
& =(1+J C) R \xi .
\end{aligned}
$$

Since $R$ maps $\mathscr{C}$ onto $\mathscr{K}$, it follows that

$$
P \mathscr{C l}=(1+J C) \mathscr{L C} \text {. }
$$

Consider next $\xi \in \mathscr{C}^{\perp}=J \mathscr{M}$, so $\xi=J \eta$ for $\eta \in \mathscr{M}$. Then

$$
2 P \xi=(R+J T) J \eta=(J(2-R)+T) \eta=(J C+1) T \eta,
$$

which is in $(1+J C) \mathscr{C l}$ since $T$, being a function of $R$, carries $\mathscr{C}$ into itself. Thus

$$
P \mathscr{C l}^{\perp} \subseteq(1+J C) \mathscr{L} \text {. }
$$

Since $\mathscr{K}=P \mathscr{C}+P \mathscr{M}^{\perp}$, it follows that

$$
\mathscr{K}=(1+J C) \mathscr{L} \text {. }
$$

We now define an isometry, $U$, from $\mathscr{H}$ to $\mathscr{C} \oplus \mathscr{C}$ by

$$
U(\xi+J \eta)=(\xi, \eta) \text { for } \xi, \eta \in \mathscr{C} \text {. }
$$

Then 


$$
U \mathscr{K}=U(1+J C) \mathscr{M}=\operatorname{graph}(C),
$$

while a similar argument shows that $U \mathscr{L}$ is the graph of $-C$.

As remarked in [7], the proof of the above result has as an almost immediate corollary one of the main results of [4], namely

Proposition 2.5 (Proposition 9 of [4]). Let $\left(\mathscr{K}_{i}, \mathscr{L}_{i}\right)$ be a pair of subspaces in general position in a Hilbert space $\mathscr{H}_{i}$, for $i=1,2$. Let $R_{i}$ be the sum of the projections on $\mathscr{K}_{i}$ and $\mathscr{L}_{i}$. Then the two pairs of subspaces are unitarily equivalent if and only if $R_{1}$ and $R_{2}$ are unitarily equivalent.

3. One real subspace. Let $\mathscr{K}$ be a (closed) real subspace of a complex Hilbert space $\mathscr{H}$ such that $\mathscr{K} \cap i \mathscr{K}=\{0\}$ and $\mathscr{K}+$ $i \mathscr{K}$ is dense in $\mathscr{H}$. In this section we will apply the results of the previous section to the pair $(\mathscr{K}, i \mathscr{K})$ of real subspaces of $\mathscr{H}$. To do this we view $\mathscr{H}$ as a real Hilbert space by equipping it with the real part of its inner-product. We can then let $P$ and $Q$ be the real linear projections on $\mathscr{K}$ and $i \mathscr{K}$ respectively, defined by means of the real inner-product on $\mathscr{H}$, and so we can define the operators $R, T$ and $J$ as in the previous section. Because of our special choice of subspaces, these operators will have additional properties:

Proposition 3.1. The operators $R$ and $T$ are complex linear and positive. Furthermore, $J$ is a conjugate linear isometry, so that $\langle J \xi, \eta\rangle=\langle J \eta, \xi\rangle$ for $\xi, \eta \in \mathscr{H}$.

Proof. Simple calculations show that $i P=Q i$, and hence that $R$ is complex linear, while $P-Q$ is conjugate linear. Then from the construction of polar decompositons $T$ will be complex linear, while $J$ is conjugate linear. We know that

$$
\operatorname{Re}\langle J \xi, \eta\rangle=\operatorname{Re}\langle\xi, J \eta\rangle=\operatorname{Re}\langle J \eta, \xi\rangle .
$$

This, together with the conjugate linearity of $J$, shows that $\langle J \xi, \eta\rangle=$ $\langle J \eta, \xi\rangle$ for $\xi, \eta \in \mathscr{H}$.

We remarked in the previous section that the modular operator, $\Delta$, of the usual Tomita-Takesaki theory is related to $R$ by $\Delta=$ $(2-R) R^{-1}$. Furthermore, a major role in that theory is played by the one-parameter unitary group $\Delta^{i t}$. Because $\mathscr{C}$ is now complex, we can expect to be able to define this group in our context also. We now show, in fact, that this can be done directly in terms of $R$. We will then derive various properties of this group, and show 
that it is characterized by the K.M.S. condition even in our setting, all this using only bounded operators.

According to Proposition 2.2 both $R$ and $2-R$ are injective, while $0 \leqq R \leqq 2$. It follows that the spectral measures for $R$ and $2-R$ are both concentrated on the open interval $(0,2)$. Now for any real $t$ the function $\lambda \mapsto \lambda^{i t}$ is well-defined, bounded and continuous on this open interval, and so by spectral theory we can define $R^{i t}$ and $(2-R)^{i t}$. Furthermore, from spectral theory we also see that in this way we obtain two commuting one-parameter unitary groups which are strongly continuous (see Lemma 3.6). From the fact that $J R J=(2-R)$ it follows easily that

$$
J R^{i t} J=(2-R)^{-i t}
$$

for all real $t$, where the minus sign in the exponent of $(2-R)$ is caused by the conjugate linearity of $J$. While we do not define the operator $\Delta$, we will nevertheless use the notation $\Delta^{i t}$ so as to conform with the usual notation of the Tomita-Takesaki theory. We define this unitary operator by

Definition 3.2. For each real $t$ let

$$
\Delta^{i t}=(2-R)^{i t} R^{-i t},
$$

so that $\left\{\Delta^{i t}\right\}$ is a one-parameter unitary group.

Proposition 3.3. For any real $t$

$$
J \Delta^{i t}=\Delta^{i t} J \text { and } \Delta^{i t} \mathscr{K}=\mathscr{L} \text {. }
$$

Proof. The first equation follows directly from the fact that $J R^{i t} J=(2-R)^{-i t}$. Now since $J^{i t}$ is a function of $R$, it commutes with $R$ and $T$, and so with $T J$. It follows that $\Delta^{i t}$ commutes with both $P$ and $Q$, and in particular that $\Delta^{i t} \mathscr{K} \subseteq \mathscr{K}$. Since this last relation is also true for $\Delta^{-i t}$, it follows that $\Delta^{i t} \mathscr{K}=\mathscr{K}$.

We can now turn to our characterization of the group $\Delta^{i t}$ in terms of the K.M.S. condition. We will not need this characterization later, but we feel that it is important to include it at this point so that it is clear that the K.M.S. property is really concerned just with real subspaces of a complex Hilbert space, and does not essentially involve the presence of von Neumann algebras.

DEFINITION 3.4. A strongly continuous one-parameter unitary group, $U_{t}$, on the Hilbert space $\mathscr{H}$ is said to satisfy the K.M.S. condition with respect to the real subspace, $\mathscr{K}$, of $\mathscr{H}$ if for any 
$\xi, \eta \in \mathscr{K}$ there is a complex-valued function, $f$, defined, bounded and continuous on the strip $-1 \leqq \operatorname{Im}(z) \leqq 0$, analytic in the interior, and with boundary values given by

$$
\begin{aligned}
f(t) & =\left\langle U_{t} \xi, \eta\right\rangle \\
f(t-i) & =\left\langle\eta, U_{t} \xi\right\rangle
\end{aligned}
$$

for all real $t$.

We remark that such a function $f$ is unique. Indeed, if there were another such function, $g$, with the same boundary conditions, the difference $f-g$ would vanish on the reals. Then the Schwartz reflection principle would yield a function analytic on the strip $-1 \leqq \operatorname{Im}(z) \leqq 1$ and also vanishing on the reals. Such a function must vanish everywhere, so that $f=g$.

The fact that

$$
f(t-i)=\left\langle\eta, U_{t} \xi\right\rangle=(f(t))^{-}
$$

makes it possible to obtain a condition which is equivalent to the K.M.S. condition but which is often easier to verify.

Proposition 3.5. A strongly-continuous one-parameter unitary group $U_{t}$ satisfies the K.M.S. condition with respect to the real subspace $\mathscr{K}$ of $\mathscr{H}$ if and only if for any $\xi, \eta \in \mathscr{K}$ there is a function, $f$, defined, bounded and continuous on the strip $-1 / 2 \leqq$ $\operatorname{Im}(z) \leqq 0$, analytic in the interior and such that

$$
f(t)=\left\langle U_{t} \xi, \eta\right\rangle \text { for all real } t
$$

and

$$
f(t-i / 2) \text { is real for all real } t \text {. }
$$

Proof. That these conditions imply the K.M.S. condition is seen by simply applying the Schwartz reflection principle along the line $\operatorname{Im}(z)=-1 / 2$. Conversely, suppose the K.M.S. conditions are satisfied. If $f$ is a "K.M.S.-function" as in Definition 3.4 such that $f(t-i)=(f(t))^{-}$, then we can define another function, $g$, on the strip $-1 \leqq \operatorname{Im}(z) \leqq 0$ by

$$
g(z)=(f(\bar{z}-i))^{-} .
$$

Then $g$ will satisfy the same properties and have the same boundary values as $f$, and so by the uniqueness remarked above we will have $g=f$. Then

$$
f(t-i / 2)=g(t-i / 2)=(f(t-i / 2))^{-},
$$

so that $f$ is real on the line $\operatorname{Im}(z)=-1 / 2$. 
This second version of the K.M.S. condition was inspired by Combes' treatment of the K.M.S. condition for weights [2].

We will now begin to show that for a given real subspace $\mathscr{K}$, the group $d^{i t}$ defined earlier satisfies the K.M.S. condition with respect to $\mathscr{K}$. For this we need the following lemma, which will also be used in later sections.

LEMMA 3.6. For any complex $z$ with $\operatorname{Im}(z) \leqq 0$ we can define the bounded operator $R^{i z}$. The function $z \mapsto R^{i z}$ is strong-operator continuous on $\operatorname{Im}(z) \leqq 0$, is analytic on $\operatorname{Im}(z)<0$, and is uniformly bounded on horizontal strips of finite width. Similar results are true for $(2-R)^{i z}$.

Proof. For any complex $z$ with $\operatorname{Im}(z) \leqq 0$ the function $\lambda \rightarrow \lambda^{i z}$ is bounded and continuous on the open interval $(0,2)$ on which the spectral measure of $R$ is supported, and so we can form $R^{i z}$. Since $\left|\lambda^{i z}\right| \leqq 2^{-\operatorname{Im}(z)}$ if $\lambda \in(0,2)$ and $\operatorname{Im}(z) \leqq 0$, it follows that $R^{i z}$ is uniformly bounded on horizontal strips of finite width. Now let $\xi \in \mathscr{H}$, and let $\left\{E_{\varepsilon}\right\}$ be the spectral resolution for $R$. Then the restriction of $R$ to $\left(1-E_{\varepsilon}\right) \mathscr{H}$ has its spectrum in $(\varepsilon, 2)$, and so has bounded logarithm. It follows that the function $R^{i z}\left(1-E_{\varepsilon}\right) \xi$ is actually analytic in the entire complex plane. Now $\left(1-E_{\varepsilon}\right) \xi$ converges to $\xi$ as $\varepsilon$ goes to 0 , since $R$ is injective. Thus $R^{i z}\left(1-E_{\varepsilon}\right) \xi$ converges to $R^{i z} \xi$, in fact uniformly on horizontal strips where $\operatorname{Im}(z) \leqq 0$, since $R^{i z}$ is uniformly bounded there. It follows that $R^{i z} \xi$ is continuous for $\operatorname{Im}(z) \leqq 0$, and analytic for $\operatorname{Im}(z)<0$.

Proposition 3.7. The one-parameter unitary group $\Delta^{i t}$ defined in Definition 3.3 satisfies the K.M.S. condition with respect to $\mathscr{K}$.

Proof. Let $\xi, \eta \in \mathscr{K}$. We would like to set $f(z)=\left\langle\Delta^{i z} \xi, \eta\right\rangle$ but this is undefined. However, we can take advantage of the fact that $\xi \in \mathscr{C}$ to extend $\Delta^{i t} \xi$ analytically to the strip $-1 / 2 \leqq \operatorname{Im}(z) \leqq 0$. For

$$
2 \xi=2 P \xi=(R+T J) \xi=R^{1 / 2}\left(R^{1 / 2}+(2-R)^{1 / 2} J\right) \xi,
$$

so that

$$
\xi=R^{1 / 2} \zeta \quad \text { if } \quad 2 \zeta=\left(R^{1 / 2}+(2-R)^{1 / 2} J\right) \xi
$$

Thus

$$
\Delta^{i t} \xi=\Delta^{i t} R^{1 / 2 \zeta}=(2-R)^{i t} R^{-i t+1 / 2 \zeta},
$$

and so we can set

$$
f(z)=\left\langle(2-R)^{i z} R^{-i z+1 / 2 \zeta}, \eta\right\rangle .
$$

From Lemma 3.6 together with the fact that multiplication of oper- 
ators is strongly continuous on bounded sets, we see that the function is defined, bounded and continuous on the strip $-1 / 2 \leqq \operatorname{Im}(z) \leqq 0$, and analytic in the interior. It is clear that

$$
f(t)=\left\langle\Delta^{i t} \xi, \eta\right\rangle \text {. }
$$

It remains to show that $f(t-i / 2)$ is real. But

$$
\begin{aligned}
f(t-i / 2) & =\left\langle(2-R)^{i t}(2-R)^{1 / 2} R^{-i t} \zeta, \eta\right\rangle \\
& =\left\langle\Delta^{i t}(2-R)^{1 / 2} \zeta, \eta\right\rangle=\left\langle(2-R)^{1 / 2} \zeta, \Delta^{-i t} \eta\right\rangle,
\end{aligned}
$$

while

$$
\begin{aligned}
2(2-R)^{1 / 2} \zeta & =(2-R)^{1 / 2}\left(R^{1 / 2}+(2-R)^{1 / 2} J\right) \xi \\
& =(T+J R) \xi=J(T J+R) \xi=2 J P \xi=2 J \xi .
\end{aligned}
$$

Thus

$$
f(t-i / 2)=\left\langle J \xi, \Delta^{-i t} \eta\right\rangle,
$$

which is real, since $J \xi \in i \mathscr{K}^{\perp}$ by Proposition 2.3 while $\Delta^{-i t} \eta \in \mathscr{K}$.

THEOREM 3.8. The group $\Delta^{i t}$ is the unique strongly continuous one-parameter group of unitaries on $\mathscr{H}$ which carries $\mathscr{K}$ onto $\mathscr{K}$ and satisfies the K.M.S. condition with respect to $\mathscr{K}$.

Proof. Let $U_{t}$ be a strongly continuous one-parameter group of unitaries on $\mathscr{H}$ which carries $\mathscr{K}$ onto $\mathscr{K}$ and satisfies the K.M.S. condition with respect to $\mathscr{K}$. We will show that $U_{t}=\Delta^{i t}$ for all $t$.

Recall that $\eta \in \mathscr{H}$ is said to be an entire vector for $\left\{U_{t}\right\}$ if there is an entire $\mathscr{H}$-valued function, $h$, such that $h(t)=U_{t} \eta$ for all real $t$. Such vectors are dense in $\mathscr{H}$. We recall the proof so that we can see that, in fact, $\mathscr{K}$ will contain a collection of entire vectors dense in $\mathscr{K}$. For $\eta \in \mathscr{K}$ let

$$
\eta_{n}=(n / \pi)^{1 / 2} \int_{-\infty}^{\infty} e^{-n t^{2}} U_{t} \eta d t
$$

Then $\eta_{n} \in \mathscr{K}$ since $\exp \left(-n t^{2}\right)$ is real and since $\mathscr{K}$ is closed and is carried into itself by $U_{t}$. It is easily seen that $\left\{\eta_{n}\right\}$ converges to $\eta$. Finally, each $\eta_{n}$ is an entire vector, since

$$
h_{n}(z)=(n / \pi)^{1 / 2} \int_{-\infty}^{\infty} e^{-n(t-z)^{2}} U_{t} \eta d t
$$

is entire, and $h_{n}(s)=U_{s} \eta_{n}$ for all real $s$.

Now let $\eta$ be an entire vector for $\left\{U_{t}\right\}$ in $\mathscr{K}$, and let $h$ be the entire function such that $h(t)=U_{t} \eta$ for real $t$. Then for fixed real 
$t$ the functions $h(t+i z)$ and $U_{t} h(i z)$ are both entire, and agree for purely imaginary $z$. They are thus equal, and so in particular

$$
h(t+i s)=U_{t} h(i s)
$$

for all real $s$ and $t$. Since $U_{t}$ is unitary, this makes it clear, in particular, that $h$ is bounded on horizontal strips of finite width.

Let $\xi$ be another vector in $\mathscr{K}$. We would like to show that

$$
\left\langle U_{t} \eta, \Delta^{i t} J \xi\right\rangle=\langle\eta, J \xi\rangle,
$$

for this will then imply that $U_{t}=\Delta^{i t}$ since $J \mathscr{K}$ and $\mathscr{K}$ are total in $\mathscr{H}$. To show this we must extend this function analytically by means of the function $h$ defined above, and by the device used in the proof of Proposition 3.7. Specifically, define $\zeta$ as in that proof so that $\xi=R^{1 / 2 \zeta}$, and set

$$
g(z)=\left\langle h(z), J(2-R)^{i z} R^{-i z+1 / 2} \zeta\right\rangle .
$$

Then, as in the proof of Proposition 3.7, $g$ is defined, bounded and continuous on the strip $-1 / 2 \leqq \operatorname{Im}(z) \leqq 0$, and analytic inside. Furthermore,

$$
g(t)=\left\langle U_{t} \eta, J \Delta^{i t} R^{1 / 2} \zeta\right\rangle=\left\langle U_{t} \eta, J \Delta^{i t} \xi\right\rangle,
$$

which is real since $U_{t} \eta \in \mathscr{K}$ while $J \Delta^{i t} \xi \in i \mathscr{K}^{\perp}$ by Proposition 2.3.

On the other hand

$$
\begin{aligned}
g(t-i / 2) & =\left\langle h(t-i / 2), J \Delta^{i t}(2-R)^{1 / 2} \zeta\right\rangle \\
& =\left\langle h(t-i / 2), \Delta^{i t} \xi\right\rangle,
\end{aligned}
$$

since $(2-R)^{1 / 2} \zeta=J \xi$ as seen in the proof of Proposition 3.7. Now if the K.M.S. condition for $\left\{U_{t}\right\}$ is applied to the pair $\left(\eta, \Delta^{i s} \xi\right)$, we obtain a function, $f$, defined, bounded and continuous on the strip and analytic on the interior such that $f(t-i / 2)$ is real and

$$
f(t)=\left\langle U_{t} \eta, \Delta^{i s} \xi\right\rangle=\left\langle h(t), \Delta^{i s} \xi\right\rangle
$$

for real $t$. Thus $f$ agrees on the $x$-axis with the entire function $\left\langle h(z), \Delta^{i s} \xi\right\rangle$, and so must agree with it everywhere on the strip. It follows that $\left\langle h(z), \Delta^{i s} \xi\right\rangle$ is real for $z=t-i / 2$. In particular this must be true for $s=t$, so that $g(t-i / 2)$ is real for all real $t$.

Thus the function $g$ is defined, continuous and bounded on the strip $-i / 2 \leqq \operatorname{Im}(z) \leqq 0$, analytic in the interior, and real-valued on the boundary of the strip. But such a function must be constant, because repeated applications of the Schwartz reflection principle will yield an extension to a bounded entire function. Thus

$$
\left\langle U_{t} \eta, \Delta^{i t} J \xi\right\rangle=\langle\eta, J \xi\rangle,
$$


so that $U_{t}=\Delta^{i t}$ for the reasons indicated above.

In fact, the following stronger form of the uniqueness property can be obtained. (We are indebted to J. Naudts for this result.)

THEOREM 3.9. Let $U_{t}$ be a strongly continuous one-parameter group of unitaries on a Hilbert space $\mathscr{H}$. Suppose that $\mathscr{K}_{0}$ is a real submanifold of $\mathscr{H}$ such that $U_{t}$ satisfies the K.M.S. condition for $\mathscr{K}_{0}$. Then $U_{t}$ also satisfies the K.M.S. condition with respect to the smallest real subspace, $\mathscr{K}$, invariant under $U_{t}$ and containing $\mathscr{K}_{0}$. Furthermore $\mathscr{K} \cap i \mathscr{K}=\{0\}$, so that if we let $\mathscr{H}_{1}$ denote the closure of $\mathscr{K}+i \mathscr{K}$, then we can define $\Delta^{i t}$ on $\mathscr{H}_{1}$ using $\mathscr{K}$. Then $\mathscr{H}_{1}$ is invariant under $U_{t}$, and $U_{t}=\Delta^{i t}$ on $\mathscr{H}_{1}$.

Proof. Let $\mathscr{K}_{1}$ denote the set of vectors $\xi \in \mathscr{X}$ such that for every $\eta \in \mathscr{K}_{0}$ there is a K.M.S. function for the pair $(\xi, \eta)$ as in Definition 3.4. Then $\mathscr{K}_{1}$ is obviously a real submanifold of $\mathscr{H}$, and $\mathscr{K}_{0} \subseteq \mathscr{K}_{1}$.

Let $\xi \in \mathscr{K}_{1}$ and $\eta \in \mathscr{K}_{0}$, and let $f$ be the K.M.S. function for the pair $(\xi, \eta)$. For fixed real $s$ define $g$ on the strip $-1 / 2 \leqq \operatorname{Im}(z) \leqq 0$ by $g(z)=f(z+s)$. Then

$$
g(t)=f(t+s)=\left\langle U_{t}\left(U_{s} \xi\right), \eta\right\rangle,
$$

and $g(t-i / 2)$ is real, so that $g$ is a K.M.S. function of the pair $\left(U_{s} \xi, \eta\right)$. Thus $\mathscr{K}_{1}$ is invariant under $U_{t}$.

We claim that $\mathscr{K}_{1}$ is also closed. Indeed let $\xi_{n}$ be a sequence in $\mathscr{K}_{1}$, let $\eta \in \mathscr{K}_{0}$ and let $f_{n}$ be the K.M.S. function (on $-1 \leqq$ $\operatorname{Im}(z) \leqq 0)$ for the pair $\left(\xi_{n}, \eta\right)$. Assume that $\xi_{n}$ converges to $\xi \in \mathscr{H}$. Applying the maximum modulus principle on the strip (p. 245 of [11]) to the function $f_{n}-f_{m}$, we find that

$$
\left|f_{n}(z)-f_{m}(z)\right| \leqq\left\|\xi_{n}-\xi_{m}\right\|\|\eta\|,
$$

so that $\left\{f_{n}\right\}$ is a uniformly Cauchy sequence on the strip. Thus this sequence converges uniformly to a function, $f$, which is defined, bounded and continuous on the strip and analytic on the interior. Furthermore

$$
f(t)=\lim f_{n}(t)=\lim \left\langle U_{t} \xi_{n}, \eta\right\rangle=\left\langle U_{t} \xi, \eta\right\rangle,
$$

and similarly

$$
f(t-i)=(f(t))^{-} .
$$

Thus $f$ is a K.M.S. function for the pair $(\xi, \eta)$, so that $\xi \in \mathscr{K}_{1}$.

Since $\mathscr{K}_{1}$ is a real closed subspace of $\mathscr{H}$ which contains $\mathscr{K}_{0}$ 
and is invariant under $U_{t}$, it must contain $\mathscr{K}$. Thus we have shown that if $\xi \in \mathscr{K}$ and $\eta \in \mathscr{K}_{0}$ then there is a K.M.S. function for the pair $(\xi, \eta)$. But we can then repeat the arguments above to show that there is a K.M.S. function for any $\xi, \eta \in \mathscr{K}$, so that $U_{t}$ satisfies the K.M.S. condition for $\mathscr{K}$.

To complete the proof it is sufficient to show that $\mathscr{K} \cup i \mathscr{K}=$ $\{0\}$, as the rest will then follow from Theorem 3.8. Suppose now that $\xi \in \mathscr{K} \cap i \mathscr{K}$, and let $f$ be the K.M.S. function for the pair $(\xi, \xi)$. Then if will be the K.M.S. function for the pair $(i \xi, \xi)$, so that both $f$ and if are real on the line $\operatorname{Im}(z)=-1 / 2$. Thus $f$ vanishes on this line and so everywhere, and so $0=f(0)=\langle\xi, \xi\rangle$, so that $\xi=0$.

We remark that the condition that $U_{t}$ carry $\mathscr{K}$ into $\mathscr{K}$ cannot be dropped in Theorem 3.8. For otherwise it would follow from Theorem 3.9 that every subspace $\mathscr{K}_{0}$ of $\mathscr{K}_{\mathscr{K}}$ such that $\mathscr{K}_{0}+i \mathscr{K}_{0}$ is dense would define the same group $\Delta^{i t}$, which is impossible.

We also remark that the more general characterization whose proof begins in the bottom paragraph of page 239 of [8] can presumably be carried over to the present setting.

Finally we confess that while it is our feeling that $J$ and $\Delta^{i t}$ are giving information about the relative positions of $\mathscr{K}$ and $i \mathscr{K}$, it is not clear to us in what sense this is really true. In particular, the characterization of $\Delta^{i t}$ by means of the K.M.S. condition can hardly be called "geometric" and it would be nice to have a characterization which was more geometric.

4. von Neumann algebras. In this section we give a proof of the main results of Tomita-Takesaki theory for the case of a von Neumann algebra with a cyclic and separating vector. The proof we give is obtained essentially by reformulating the proof given in [19] in terms of the bounded operators which we studied in the previous two sections.

Let $M$ be a von Neumann algebra acting on a Hilbert space $\mathscr{H}$, and let $\omega$ be a cyclic and separating vector for $M$. Let $M^{\prime}$ denote the commutant of $M$, and let $M_{s}$ and $M_{s}^{\prime}$ denote the set of self-adjoint elements in $M$ and $M^{\prime}$ respectively.

Proposition 4.1. Let $\mathscr{K}$ denote the closure of $M_{s} \omega$. Then $\mathscr{K}$ is a (closed) real subspace of $\mathscr{H}$ such that $\mathscr{K} \cap i \mathscr{K}=\{0\}$ and $\mathscr{K}+i \mathscr{K}$ is dense in $\mathscr{H}$. Moreover $M_{s}^{\prime} \omega \subseteq i \mathscr{K}^{\perp}$.

Proof. Clearly $\mathscr{K}+i \mathscr{K} \supseteq M \omega$, and so is dense since $\omega$ is cyclic. We know that $M_{s} \omega \cap i M_{s} \omega=\{0\}$ because $\omega$ is separating, 
but this does not imply that $\mathscr{K} \cap i \mathscr{K}=\{0\}$ since $\mathscr{K}$ can be strictly bigger than $M_{s} \omega$. But let us first show that $M_{s}^{\prime} \omega \subseteq i \mathscr{C}^{\perp}$. If $x \in M_{\mathrm{s}}$ and $x^{\prime} \in M_{s}^{\prime}$, then

$$
\left\langle x \omega, x^{\prime} \omega\right\rangle=\left\langle x^{\prime} x \omega, \omega\right\rangle=\left\langle x^{\prime} \omega, x \omega\right\rangle,
$$

so that $\left\langle x \omega, x^{\prime} \omega\right\rangle$ is real, and

$$
\operatorname{Re}\left\langle x \omega, i x^{\prime} \omega\right\rangle=0 .
$$

Thus $x^{\prime} \omega \in i \mathscr{K}^{\perp}$ and $M_{s}^{\prime} \omega \subseteq i \mathscr{K}^{\perp}$. But then $M^{\prime} \omega \subseteq i \mathscr{K}^{\perp}+\mathscr{K}^{\perp} \subseteq$ $(\mathscr{K} \cap i \mathscr{K})^{\perp}$. Since $\omega$ is cyclic for $M^{\prime}$, it follows that $(\mathscr{K} \cap i \mathscr{K})^{\perp}=$ $\mathscr{H}$, so that $\mathscr{K} \cap i \mathscr{K}=\{0\}$.

One can, in fact, show that $\left(M_{s}^{\prime} \omega\right)^{-}=i \mathscr{K}^{\perp}$ (see Theorem 1 of [10]), but we will not need this result here.

Since the above proposition says that $\mathscr{K}$ as defined above satisfies the nondegeneracy conditions used in the previous sections, we can use $\mathscr{K}$ to define operators $R$ and $T$, and especially $J$ and $\Delta^{i t}$. The main results of the Tomita-Takesaki theory are:

THEOREM 4.2. If $\mathscr{K}$ is defined in terms of $M$ as above, and if $J$ and $\Delta^{\text {it }}$ are defined by $\mathscr{K}$, then

(1) $J M J=M^{\prime}$,

(2) $\Delta^{i t} M \Delta^{-i t}=M$ for all real $t$.

The main difficulty in proving the above theorem seems to stem from the paucity of information we have relating the subspace $\mathscr{K}$ to the action of the elements of $M$ on $\mathscr{H}$. In fact, the only such piece of information we seem to have is the following lemma, whose proof is obtained by modifying the proof of Sakai's linear RadonNikodym theorem [12].

Lemma 4.3. Let $x^{\prime} \in M_{s}^{\prime}$. Then for any $\lambda \in C$ with $\operatorname{Re}(\lambda)>0$ there is a unique $x \in M_{s}$ such that

$$
\left\langle y \omega, x^{\prime} \omega\right\rangle=\operatorname{Re}(\lambda\langle y \omega, x \omega\rangle) \text { for all } y \in M_{s} .
$$

Proof. By real linearity we may assume that $\operatorname{Re}(\lambda)=1$ and that $0 \leqq x^{\prime} \leqq 1$. Define normal functionals $\psi$ and $\psi_{x}$ on $M$ for any $x \in M_{s}$ by

$$
\psi(y)=\left\langle y \omega, x^{\prime} \omega\right\rangle, \quad \psi_{x}(y)=\operatorname{Re}(\lambda\langle y \omega, x \omega\rangle)
$$

for $y \in M_{s}$. Clearly $\psi$ and $\psi_{x}$ are in the self-adjoint part of the predual of $M$. What we need to show is that there is some $x \in M_{s}$ such that $\psi=\psi_{x}$. Let 


$$
V=\left\{\psi_{x}: x \in M_{s} \text { and }\|x\| \leqq 1\right\} .
$$

Since $x \mapsto \psi_{x}$ is clearly continuous for the $w^{*}$-topology on $M$ and the weak topology on the predual of $M$, it follows that $V$ is weakly compact. Also $V$ is clearly convex. We need to show that $\psi \in V$. Suppose that $\psi \notin V$. Then by the Hahn-Banach separation theorem there is an $h \in M_{s}$ such that

$$
\psi_{x}(h)<\psi(h) \text { for all } x \in M_{s},\|x\| \leqq 1 .
$$

This means that

$$
\operatorname{Re}(\lambda\langle h \omega, x \omega\rangle)<\left\langle h \omega, x^{\prime} \omega\right\rangle
$$

for all $x \in M_{8},\|x\| \leqq 1$. Now let $h=u|h|$ be the polar decomposition of $h$. Since $h$ is self-adjoint so is $u$, and $u$ and $|h|$ commute. Let $x=u$. Then

$$
\begin{aligned}
& \operatorname{Re}(\lambda\langle h \omega, u \omega\rangle)\left\langle\left\langle h \omega, x^{\prime} \omega\right\rangle=\left\langle h\left(x^{\prime}\right)^{1 / 2} \omega,\left(x^{\prime}\right)^{1 / 2} \omega\right\rangle\right. \\
& \leqq\left\langle|h|\left(x^{\prime}\right)^{1 / 2} \omega,\left(x^{\prime}\right)^{1 / 2} \omega\right\rangle=\left\langle|h| x^{\prime} \omega, \omega\right\rangle \\
& \leqq\langle|h| \omega, \omega\rangle=\langle h \omega, u \omega\rangle=\operatorname{Re}(\lambda\langle h \omega, u \omega\rangle)
\end{aligned}
$$

(since $\operatorname{Re}(\lambda)=1$ ). This is a contradiction.

CoROLlary 4.4. For any $x^{\prime} \in M^{\prime}$ there is an $x \in M$ such that

$$
J T x^{\prime} \omega=x \omega, \quad J T x^{*} \omega=x^{*} \omega .
$$

Proof. Suppose first that $x^{\prime} \in M_{s}^{\prime}$. If we apply Lemma 4.3 with $\lambda=1$ we obtain an $x \in M_{s}$ such that

$$
\operatorname{Re}\langle y \omega, x \omega\rangle=\left\langle y \omega, x^{\prime} \omega\right\rangle \text { for all } y \in M_{s} .
$$

This means that $x \omega$ is the orthogonal projection of $x^{\prime} \omega$ onto $\mathscr{K}$ (for the real inner product), that is, $P x^{\prime} \omega=x \omega$. Now $x^{\prime} \omega \in i \mathscr{C}^{\perp}$, so that $Q x^{\prime} \omega=0$. Thus

$$
J T x^{\prime} \omega=(P-Q) x^{\prime} \omega=x \omega .
$$

The general result now follows by conjugate linearity.

Lemma 4.5. Let $x^{\prime} \in M^{\prime}$. Then for any $\lambda \in C$ with $\operatorname{Re}(\lambda)>0$ there is an $x \in M$ such that

$$
T J x^{\prime} J T=\lambda(2-R) x R+\bar{\lambda} R x(2-R) .
$$

Proof. By linearity we may assume that $x^{\prime} \in M_{s}^{\prime}$. Then according to Lemma 4.3 (with $x$ replaced by $x / 2$ ) there is an $x \in M_{s}$ such that 


$$
\left\langle y \omega, x^{\prime} \omega\right\rangle=\lambda\langle y \omega, x \omega\rangle+\bar{\lambda}\langle x \omega, y \omega\rangle
$$

for all $y \in M_{s}$. It is clear that this relation will hold for arbitrary $y \in M$ if we change the third $y$ to $y^{*}$. If we then substitute $z^{*} y$ for $y$, where $y, z \in M$, we obtain

$$
\left\langle y \omega, x^{\prime} z \omega\right\rangle=\lambda\langle y \omega, z x \omega\rangle+\bar{\lambda}\langle y x \omega, z \omega\rangle .
$$

Now let $y^{\prime}$ and $z^{\prime}$ be arbitrary elements of $M$. By Corollary 4.4 there exist $y, z \in M$ such that

$$
J T y^{\prime} \omega=y \omega, \quad J T z^{\prime} \omega=z \omega .
$$

Substituting these expressions for $y \omega$ and $z \omega$ in the above equation and rearranging, using Proposition 3.1, we obtain.

$$
\left\langle z^{\prime} \omega, T J x^{\prime} J T y^{\prime} \omega\right\rangle=\lambda\left\langle T J z x \omega, y^{\prime} \omega\right\rangle+\bar{\lambda}\left\langle z^{\prime} \omega, T J y x \omega\right\rangle .
$$

Now for any $u \in M_{s}$ we have $P u \omega=u \omega$, so that

$$
T J u \omega=(P-Q) u \omega=(2-P-Q) u \omega=(2-R) u \omega .
$$

Similarly for $u^{\prime} \in M_{\mathrm{s}}^{\prime}$ we have $Q u^{\prime} \omega=0$ so that

$$
T J u^{\prime} \omega=(P-Q) u^{\prime} \omega=(P+Q) u^{\prime} \omega=R u^{\prime} \omega .
$$

Using the fact that $R$ is linear while $T J$ is conjugate-linear, we see that

$$
T J u \omega=(2-R) u^{*} \omega, \quad T J u^{\prime} \omega=R u^{\prime *} \omega
$$

for any $u \in M$ and $u^{\prime} \in M^{\prime}$. It follows from this, Corollary 4.4 and $x^{*}=x$, that

$$
\begin{aligned}
T J z x \omega & =(2-R) x^{*} z^{*} \omega=(2-R) x J T z^{*} \omega \\
& =(2-R) x R z^{\prime} \omega .
\end{aligned}
$$

Similarly $T J y x \omega=(2-R) x R y^{\prime} \omega$. Substituting these expressions in (1), we obtain

$$
\left\langle z^{\prime} \omega, T J x^{\prime} J T y^{\prime} \omega\right\rangle=\lambda\left\langle(2-R) x R z^{\prime} \omega, y^{\prime} \omega\right\rangle+\bar{\lambda}\left\langle z^{\prime} \omega,(2-R) x R y^{\prime} \omega\right\rangle,
$$

Since $\omega$ is cyclic for $M^{\prime}$, and $y^{\prime}$ and $z^{\prime}$ are arbitrary elements of $M^{\prime}$, we obtain the desired equation.

We will now "solve" the operator equation obtained in the above lemma for $x$ in terms of $x^{\prime}$. We could do this by reformulating the proof in [19]. But instead we will take into account an argument of U. Haagerup which makes the proof slightly more direct. This is based on the following easy lemma. 
LEMMA 4.6. Let $\lambda=e^{i \phi / 2}$ where $-\pi<\phi<\pi$. Let $f$ be a complex-valued function defined, bounded and continuous on the strip $|\operatorname{Re}(z)| \leqq 1 / 2$ and analytic inside this strip. Then

$$
f(0)=\int_{-\infty}^{+\infty} e^{-\dot{\varphi} t}\left(e^{\pi t}+e^{-\pi t}\right)^{-1}(\lambda f(i t+1 / 2)+\bar{\lambda} f(i t-1 / 2)) d t .
$$

Proof. Define $g(z)=\pi e^{i \dot{\phi} z}(\sin \pi z)^{-1} f(z)$. Then $g$ has a simple pole at $z=0$ with residue $f(0)$. Furthermore $g$ tends to zero at infinity within the strip because $f$ is bounded and $-\pi<\phi<\pi$. Therefore we can apply Cauchy's integral formula, integrating along the boundary to obtain

$$
f(0)=(2 \pi i)^{-1}\left[\int_{-\infty}^{+\infty} g(i t+1 / 2) i d t-\int_{-\infty}^{+\infty} g(i t-1 / 2) i d t\right] .
$$

Now $\sin \pi(i t+1 / 2)=\cos \pi i t=\left(e^{\pi t}+e^{-\pi t}\right) / 2$, while

$$
e^{i \dot{\phi}(i t+1 / 2)}=\lambda e^{-\phi t} \quad \text { and } \quad e^{i \dot{\phi}(i t-1 / 2)}=\bar{\lambda} e^{-\phi t},
$$

and from this the desired formula follows.

LEMMA 4.7. Let $\lambda=e^{i \dot{\phi} / 2}$ where $-\pi<\phi<\pi$, and let $x$ and $x^{\prime}$ be as in Lemma 4.5. Then

$$
x=\int_{-\infty}^{+\infty} e^{-\phi t}\left(e^{\pi t}+e^{-\pi t}\right)^{-1} \Delta^{i t} J x^{\prime} J \Delta^{-i t} d t .
$$

Proof. Let $\xi, \eta \in \mathscr{H}$, and define $f$ for $|\operatorname{Re}(z)| \leqq 1 / 2$ by

$$
f(z)=\left\langle R^{-z+1 / 2}(2-R)^{z+1 / 2} x R^{z+1 / 2}(2-R)^{-z+1 / 2} \xi, \eta\right\rangle .
$$

Then from Lemma 3.6 it follows that $f$ satisfies the requirements of Lemma 4.6. Moreover

$$
\begin{aligned}
& \lambda f(i t+1 / 2)=\lambda\left\langle\Delta^{i t}(2-R) x R \Delta^{-i t} \xi, \eta\right\rangle \\
& \bar{\lambda} f(i t-1 / 2)=\bar{\lambda}\left\langle\Delta^{i t} R x(2-R) \Delta^{-i t} \xi, \eta\right\rangle,
\end{aligned}
$$

so that from Lemma 4.5

$$
\lambda f(i t+1 / 2)+\bar{\lambda} f(i t-1 / 2)=\left\langle\Delta^{i t} T J x^{\prime} J T \Delta^{-i t} \xi, \eta\right\rangle .
$$

On the other hand

$$
f(0)=\left\langle R^{1 / 2}(2-R)^{1 / 2} x R^{1 / 2}(2-R)^{1 / 2} \xi, \eta\right\rangle=\langle T x T \xi, \eta\rangle .
$$

Thus from Lemma 4.6 we obtain

$$
\langle T x T \xi, \eta\rangle=\int_{-\infty}^{+\infty} e^{-\phi t}\left(e^{\pi t}+e^{-\pi t}\right)^{-1}\left\langle\Delta^{i t} T J x^{\prime} J T \Delta^{-i t} \xi, \eta\right\rangle d t .
$$


The desired equation now follows from the fact that $T$ is injective and so has dense range.

We can now come close to proving the main theorem.

LEMMA 4.8. For every $x^{\prime} \in M^{\prime}$ and every real $t$ we have

$$
\Delta^{i t} J x^{\prime} J \Delta^{-i t} \in M \text {. }
$$

Proof. Let $x \in M$ be related to $x^{\prime}$ as in Lemma 4.5 , and let $y^{\prime}$ be any element of $M^{\prime}$. Then $y^{\prime}$ commutes with the right hand side of the equation of Lemma 4.7. Thus if $\xi, \eta \in \mathscr{H}$ and if we define $g$ by

$$
g(t)=\left\langle y^{\prime} \Delta^{i t} J x^{\prime} J \Delta^{-i t} \xi, \eta\right\rangle-\left\langle\Delta^{i t} J x^{\prime} J \Delta^{-i t} y^{\prime} \xi, \eta\right\rangle,
$$

then we have

$$
\int_{-\infty}^{+\infty} e^{-\phi t}\left(e^{\pi t}+e^{-\pi t}\right)^{-1} g(t) d t=0
$$

for all $\phi$ with $-\pi<\phi<\pi$. But then $g$ itself must vanish. To see this, let

$$
f(z)=\int_{-\infty}^{+\infty} e^{-z t}\left(e^{\pi t}+e^{-\pi t}\right)^{-1} g(t) d t
$$

for $|\operatorname{Re}(z)|<\pi$. Then in this region $f$ will be analytic. But it vanishes for real $z$, and so vanishes everywhere. In particular it vanishes for $z=i s$ for any real $s$. But then from the injectivity of the Fourier transform it follows that $g$ vanishes. Since this is true for all $\xi, \eta \in \mathscr{H}$, we obtain the desired result.

Setting $t=0$, we see in particular that $J M^{\prime} J \subseteq M$. To complete the proof of the main theorem it is sufficient to verify that $J M J \subseteq M^{\prime}$. One way to verify this is to show that the $J$ obtained by interchanging the roles of $M$ and $M^{\prime}$ is the same as the $J$ used above. It is easily seen that this is true if $\left(M_{s}^{\prime} \omega\right)^{-}=i \mathscr{K}^{\perp}$, and this latter follows from Theorem 1 of [10]. However, we give now a more direct verification of the fact that $J M J \subseteq M^{\prime}$.

LEMMA 4.9. $J M J \subseteq M^{\prime}$.

Proof. Notice first that $J \omega=\omega$. This is because $\omega \in \mathscr{K} \cap i \mathscr{K}^{\perp}$, so that $P \omega=\omega$ and $Q \omega=0$, so that $R \omega=\omega=T J \omega$ and $T \omega=\omega$.

Now we know that $J \mathscr{K}=i \mathscr{K}^{\perp}$ and $M_{s} \omega \subseteq \mathscr{K}$, so that $\langle J x \omega, y \omega\rangle$ is real for all $x, y \in M_{s}$. Then

$$
\langle J x \omega, y \omega\rangle=\langle y \omega, J x \omega\rangle=\langle x \omega, J y \omega\rangle
$$


from Proposition 3.1. Since $J \omega=\omega$, this can be rewritten as

$$
\langle y(J x J) \omega, \omega\rangle=\langle\omega, x(J y J) \omega\rangle .
$$

But this last equation is linear in both $x$ and $y$, and so holds actually for all $x, y \in M$. Then we can replace $y$ by $y\left(J y^{\prime} J\right)$ for any $y^{\prime} \in M^{\prime}$, since we know that $J y^{\prime} J \in M$. We obtain

$$
\left\langle y\left(J y^{\prime} J\right)(J x J) \omega, \omega\right\rangle=\left\langle\omega, x J y J y^{\prime} J J \omega\right\rangle .
$$

Assuming still that $x, y \in M_{s}$, we find that the left hand side becomes

$$
\begin{aligned}
\left\langle y J y^{\prime} x \omega, \omega\right\rangle & =\left\langle J(J y J) x y^{\prime} \omega, \omega\right\rangle=\left\langle J \omega,(J y J) x y^{\prime} \omega\right\rangle \\
& =\left\langle x(J y J) w, y^{\prime} \omega\right\rangle .
\end{aligned}
$$

while the right hand side becomes $\left\langle(J y J) x \omega, y^{\prime} \omega\right\rangle$. Since this is true for all $y^{\prime} \in M^{\prime}$, and $\omega$ is cyclic for $M^{\prime}$, we have

$$
x(J y J) \omega=(J y J) x \omega
$$

for all $x, y \in M_{s}$. By linearity this actually holds for all $x, y \in M$. Replacing $x$ by $x z$ for $z \in M$, we obtain

$$
(J y J) x z \omega=x z J y J \omega=x(J y J) z \omega .
$$

Since this is true for all $z \in M$ and $\omega$ is cyclic for $M$, it follows that

$$
(J y J) x=x(J y J),
$$

so that $J y J \in M^{\prime}$ as desired.

Proof of Theorem 4.2. From Lemmas 4.8 and 4.9 it follows that $J M J=M^{\prime}$, and so $J M^{\prime} J=M$. Then from Lemma 4.8 it follows that $\Delta^{i t} M \Delta^{-i t}=M$.

As an easy consequence of Theorem 4.2 together with the results in $\S 3$ we obtain the K.M.S. property for a faithful normal state on a von Neumann algebra.

THeORem 4.10. Let $M$ be a von Neumann algebra, and let $\phi$ be a faithful normal state on $M$. Then there is a unique strongly continuous one-parameter group of *-automorphisms, $\sigma_{i}$, of $M$ that satisfies the K.M.S. condition with respect to $\phi$, that is, such that for any $x, y \in M$ there is a complex-valued function, $f$, defined, bounded and continuous on the strip $-1 \leqq \operatorname{Im}(z) \leqq 0$, analytic inside the strip, and with boundary values

$$
f(t)=\phi\left(y \sigma_{t}(x)\right), \quad f(t-i)=\dot{\phi}\left(\sigma_{t}(x) y\right)
$$


for all real $t$. If $\phi$ is of the form $\phi(x)=\langle x \omega, \omega\rangle$ where $\omega$ is $a$ cyclic and separating vector for $M$, then

$$
\sigma_{t}(x)=\Delta^{i t} x \Delta^{-i t},
$$

where $\Delta^{i t}$ is the unitary group associated to the real subspace $\mathscr{K}=$ $\left(M_{s}(\omega)^{-}\right.$as in $\S 3$.

Proof. We can assume that $\phi$ is of the form $\phi(x)=\langle x \omega, \omega\rangle$ where $\omega$ is a cyclic and separating vector for $M$. Let $\mathscr{K}=\left(M_{s} \omega\right)^{-}$, let $\Delta^{i t}$ be the one-parameter group associated with $\mathscr{K}$, and let $\sigma_{t}(x)=$ $\Delta^{i t} x \Delta^{-i t}$. Then $\sigma_{t}$ defines a strongly continuous one-parameter group of *-automorphisms of $M$ according to Theorem 4.2. Let us now apply Proposition 3.7 to $\mathscr{K}$. For any $x, y \in M_{s}$ we have the pair of vectors $(x \omega, y \omega)$ in $\mathscr{K}$, to which there corresponds a K.M.S. function $f$ on the strip $-1 \leqq \operatorname{Im}(z) \leqq 0$ such that

$$
\begin{aligned}
f(t) & =\left\langle\Delta^{i t} x \omega, y \omega\right\rangle=\left\langle\Delta^{i t} x \Delta^{-i t} \omega, y \omega\right\rangle \\
& =\left\langle y \sigma_{t}(x) \omega, \omega\right\rangle=\phi\left(y \sigma_{t}(x)\right) .
\end{aligned}
$$

(Recall that in the proof of Lemma 4.9 we saw that $R \omega=\omega$, so that $\Delta^{i t} \omega=\omega$ for all $t$.) Then

$$
f(t-i)=f(t)^{-}=\phi\left(\sigma_{t}(x) y\right) .
$$

By linearity it follows that for all $x, y \in M$ there is a K.M.S. function, $f$, on the strip such that

$$
f(t)=\phi\left(y \sigma_{t}(x)\right), \quad f(t-i)=\phi\left(\sigma_{t}(x) y\right) .
$$

That is, $\sigma_{t}$ satisfies the K.M.S. condition for $\phi$.

Conversely, let $\alpha_{t}$ be a strongly continuous one-parameter group of *-automorphisms of $M$ satisfying the K.M.S. condition for $\phi$. As is well-known, $\phi$ must be invariant under $\alpha_{t}$. (In the K.M.S. condition let $y=1$, so that $f(t)=f(t-i)$ for all $t$, so that, as before, $f$ is constant.) Then there is a one-parameter unitary group, $U_{t}$, such that $U_{t} x \omega=\alpha_{t}(x) \omega$ for all $x \in M$ and all real $t$. So for any pair $x, y \in M_{s}$ there is a K.M.S. function $f$ such that

$$
\begin{aligned}
& f(t)=\phi\left(y \alpha_{t}(x)\right)=\left\langle y \alpha_{t}(x) \omega, \omega\right\rangle=\left\langle U_{t} x \omega, y \omega\right\rangle \\
& f(t-i)=f(t)^{-} .
\end{aligned}
$$

It now follows from Theorem 3.9, with $\mathscr{K}_{0}=M_{s} \omega$, that $U_{t}=\Delta^{i t}$.

5. Left Hilbert algebras. In this section we treat the more general case of left Hilbert algebra. Since one of the axioms in the usual definition of Hilbert algebras $[13,18]$ involves unbounded 
operators, we should start by giving an equivalent definition in which this axiom has been reformulated so as not to involve unbounded operators. But because we feel that this axiom appears somewhat unnatural at first (for either approach), we prefer to introduce it gradually. This has the added advantage of making clearer at exactly what point of the development this axiom is needed.

Notation AND Assumptions 5.1. Let $\mathscr{A}$ be an involutive algebra over $C$, with involution denoted by $\xi \rightarrow \xi^{\sharp}$ for $\xi \in \mathscr{A}$. We suppose further that $\mathscr{A}$ is equipped with a scalar product, $\xi, \eta \mapsto$ $\langle\xi, \eta\rangle$ for $\xi, \eta \in \mathscr{A}$, and with the corresponding norm. We will denote the Hilbert space obtained by completing $\mathscr{A}$ for this norm by $\mathscr{H}$. We will assume that

(1) Left multiplication is continuous, that is, for any $\xi \in \mathscr{A}$ the linear operator $\zeta \rightarrow \xi \zeta$ for $\zeta \in \mathscr{A}$ is continuous, and so extends to a bounded operator on $\mathscr{H}$, denoted by $\pi(\xi)$.

(2) We have $\langle\xi \zeta, \eta\rangle=\left\langle\zeta, \xi^{\sharp} \eta\right\rangle$ for $\xi$, $\zeta, \eta \in \mathscr{A}$, so that the representation $\pi$ of $\mathscr{A}$ on $\mathscr{H}$ is a $*$-representation.

(3) The subalgebra $\mathscr{A}^{2}$ of $\mathscr{A}$, spanned by the elements $\xi \eta$ for $\xi, \eta \in \mathscr{A}$, is dense in $\mathscr{A}$, so that the representation $\pi$ is nondegenerate. We will let $\mathscr{L}(\mathscr{A})=\pi(\mathscr{A})^{\prime \prime}$, and call it the left von Neumann algebra associated with $\mathscr{A}$.

Of course $\pi(\mathscr{A})$ will be strong operator dense in $\mathscr{L}(\mathscr{A})$ by the double commutant theorem. It is a trivial matter to verify that if $M$ is a von Neumann algebra with a cyclic and separating vector $\omega$, then the set $\mathscr{A}=M \omega$ becomes an involutive algebra with the above properties if it is equipped with the *-algebra structure it inherits from $M$. Furthermore $\pi(x \omega)=x$ for all $x \in M$, so that $\mathscr{L}(\mathscr{A})=M$.

In the theory of left Hilbert algebras an important role is played by the analogue of the set $M^{\prime} \omega$. The appropriate candidate in the present setting seems to be:

Definition 5.2. Let $\mathscr{A}^{\prime}$ denote the set of vector $\eta \in \mathscr{H}$ such that there is a bounded operator, $b$, on $\mathscr{H}$ and another vector $\eta_{1} \in \mathscr{L}$ such that

$$
b \xi=\pi(\xi) \eta, \quad b^{*} \xi=\pi(\xi) \eta_{1} \text { for all } \xi \in \mathscr{A} .
$$

Clearly $b$ is unique since $\mathscr{A}$ is dense, and so we can denote it by $\pi^{\prime}(\eta)$. Furthermore $\eta_{1}$ is unique because $\pi$ is nondegenerate, so that we can denote it by $\eta^{\prime}$. Thus

$$
\pi^{\prime}(\eta) \xi=\pi(\xi) \eta, \quad \pi^{\prime}(\eta)^{*} \xi=\pi(\xi) \eta^{\prime} \quad \text { for all } \xi \in \mathscr{A} \text {. }
$$

It is not clear that $\mathscr{A}^{\prime}$ contains any nonzero vectors, and in 
fact we will have to impose an additional axiom to insure this. But we can first obtain the following important properties:

Proposition 5.3. If $\eta \in \mathscr{A}^{\prime}$ then $\eta^{b} \in \mathscr{A}^{\prime}$, and if $\eta, \zeta \in \mathscr{A}^{\prime}$ then $\pi^{\prime}(\eta) \zeta \in \mathscr{A}^{\prime}$. Thus $\mathscr{A}^{\prime}$ becomes a *-algebra with product defined by $\eta_{\circ} \zeta=\pi^{\prime}(\eta) \zeta$ and involution ${ }^{b}$. Moreover, $\pi^{\prime}$ is a *-representation of $\mathscr{A}^{\prime}$ on $\mathscr{Z}$ and $\pi^{\prime}\left(\mathscr{A}^{\prime}\right) \subseteq \mathscr{L}(\mathscr{A})^{\prime}$.

Proof. We show first that $\pi^{\prime}\left(\mathscr{A}^{\prime}\right) \subseteq \mathscr{L}(\mathscr{A})^{\prime}$. Let $\eta \in \mathscr{A}^{\prime}$ and $\xi, \zeta \in \mathscr{A}$. Then

$$
\begin{aligned}
\pi^{\prime}(\eta) \pi(\xi) \zeta & =\pi^{\prime}(\eta) \xi \zeta=\pi(\xi \zeta) \eta \\
& =\pi(\xi) \pi(\zeta) \eta=\pi(\xi) \pi^{\prime}(\eta) \zeta .
\end{aligned}
$$

Since $\mathscr{A}$ is dense and $\pi(\mathscr{A})$ generates $\mathscr{L}(\mathscr{A})$, the result follows.

From the definition of $\mathscr{C}^{\prime}$ it is clear that if $\eta \in \mathscr{C}^{\prime}$ then $\eta^{b} \in$ $\mathscr{A}^{\prime}$ and $\pi^{\prime}\left(\eta^{b}\right)=\pi^{\prime}(\eta)^{*}$, as well as that $\eta^{b b}=\eta$. Now let $\eta, \zeta \in \mathscr{A}^{\prime}$ and $\xi \in \mathscr{A}$. Then

$$
\begin{aligned}
\pi(\xi) \pi^{\prime}(\eta) \zeta & =\pi^{\prime}(\eta) \pi(\xi) \zeta=\pi^{\prime}(\eta) \pi^{\prime}(\zeta) \xi \\
\pi(\xi) \pi^{\prime}\left(\zeta^{b}\right) \eta^{b} & =\pi^{\prime}\left(\zeta^{b}\right) \pi(\xi) \eta^{b}=\pi^{\prime}(\zeta)^{*} \pi^{\prime}(\eta)^{*} \xi .
\end{aligned}
$$

From the definition of $\mathscr{A}^{\prime}$ it follows that $\pi^{\prime}(\eta) \zeta \in \mathscr{H}^{\prime}$. We also see from this that $(\eta \circ \zeta)^{b}=\zeta^{b} \circ \eta^{b}$ and that $\pi^{\prime}(\eta \circ \zeta)=\pi^{\prime}(\eta) \pi^{\prime}(\zeta)$.

To the extent that $\mathscr{A}^{\prime}$ contains nonzero elements it will also contain nonzero self-adjoint elements. Let $\eta \in \mathscr{A}^{\prime}$ with $\eta=\eta^{b}$. Then for $\xi \in \mathscr{A}$ we have

$$
\left\langle\eta, \xi^{\sharp} \xi\right\rangle=\left\langle\eta, \pi(\xi)^{*} \xi\right\rangle=\langle\pi(\xi) \eta, \xi\rangle=\left\langle\pi^{\prime}(\eta) \xi, \xi\right\rangle
$$

which is real since $\pi^{\prime}(\eta)$ is self-adjoint. If we let $\mathscr{K}$ denote the (closed) real subspace spanned by the $\xi^{\sharp} \xi$ for $\xi \in \mathscr{A}$, then the above says that $\eta \in i \mathscr{K}^{\perp}$, where we are now using the corresponding real inner product on $\mathscr{H}$. (In particular, we see that $\mathscr{H}^{\prime} \cong i \mathscr{K}^{\perp}+$ $\mathscr{K}^{+}$.) The following technical lemma shows, among other things, that conversely if $i \mathscr{K}^{\perp}$ contains nonzero vectors, then so does $\mathscr{A}^{\prime}$.

Lemma 5.4. For any $\eta \in \mathscr{C}$ there are elements $a, b, c \in \mathscr{L}(\mathscr{A})^{\prime}$ such that

(1) $0 \leqq a \leqq 1, \quad 0 \leqq c \leqq 1, \quad b c=(1-a) b$

$$
a(1-a)=b b^{*} \quad \text { and } \quad c(1-c)=b^{*} b .
$$

(2) $\pi(\xi) b \eta=(1-a) \xi, \quad \pi(\xi)(1-c) \eta=b^{*} \xi, \quad$ for $\xi \in \mathscr{A}$. If in fact $\eta \in i \mathscr{K}^{\perp}$, then

(3) $\pi(\xi) a \eta=b \xi, \quad \pi(\xi) b^{*} \eta=c \xi \quad$ for $\xi \in \mathscr{A}$. 
If $\eta \in i \mathscr{K}^{\perp}$ and $\eta \neq 0$, then $b \eta \neq 0$ and $b \eta \in \mathscr{A}^{\prime}$.

Proof. Let $\eta \in \mathscr{H}$. We use the closure of the graph of $\xi \mapsto$ $\pi(\xi) \eta$ for $\xi \in \mathscr{A}$. Thus let $\mathscr{N}$ be the subspace of $\mathscr{H} \oplus \mathscr{H}$ defined by

$$
\mathscr{N}=\{(\xi, \pi(\xi) \eta): \xi \in \mathscr{A}\}^{-}
$$

Let $E$ be the projection onto $\mathscr{N}$, and let the matrix for $E$ be

$$
E=\left(\begin{array}{ll}
a & b \\
b^{*} & c
\end{array}\right)
$$

Since $\mathscr{N}$ is invariant under the obvious action of $\mathscr{A}$ on $\mathscr{H} \oplus \mathscr{H}$, it follows that $a, b, c \in \mathscr{L}(\mathscr{A})^{\prime}$. Then all the properties (1) follow from the fact that $E$ is a projection. For any $\xi \in \mathscr{A}$ we have $(\xi, \pi(\xi) \eta) \in \mathscr{N}$, so that $E$ carries this vector to itself. Thus

$$
a \xi+b \pi(\xi) \eta=\xi, \quad b^{*} \xi+c \pi(\xi) \eta=\pi(\xi) \eta .
$$

Since $b, c \in \mathscr{L}(\mathscr{A})^{\prime}$, property (2) follows.

Suppose now that $\eta \in i \mathscr{K}^{\perp}$. Then $\left\langle\eta, \xi^{\sharp} \xi\right\rangle$ is real for all $\xi \in \mathscr{A}$, so that

$$
\langle\pi(\xi) \eta, \xi\rangle-\langle\xi, \pi(\xi) \eta\rangle=\left\langle\eta, \xi^{\ddagger} \xi\right\rangle-\left\langle\xi^{\sharp} \xi, \eta\right\rangle=0 .
$$

Then the sesquilinear form for this quadratic form in $\xi$ must also vanish, that is

$$
\langle\pi(\xi) \eta, \zeta\rangle-\langle\xi, \pi(\zeta) \eta\rangle=0 \text { for all } \xi, \zeta \in \mathscr{A} \text {. }
$$

But this says that $(\pi(\xi) \eta,-\xi) \in \mathscr{N}^{\perp}$ for $\xi \in \mathscr{A}$, so that $E$ is zero on this vector. Property (3) follows from this. Now from the first part of property (2) it already follows that $b \eta \in \mathscr{A}^{\prime}$, since $(1-a)$ is self-adjoint. But we could have $b \eta=0$. This would mean that $(1-a)=0$, so that $b=0$ from property (1). But if $\eta \in i \mathscr{K}^{\perp}$, it would then follow from property (3) that $\eta=0$.

Thus to make sure that $\mathscr{A}^{\prime}$ is large, we must make sure that $i \mathscr{K}^{\perp}$ is large. Since we also want to be able to use the results of $\S 2$, a natural way to try to do this is to assume that $\mathscr{K} \cap i \mathscr{K}=$ $\{0\}$. Notice that even before assuming this we know by polarization that $\mathscr{K}+i \mathscr{K}$ contains all elements of the form $\xi \zeta$ for $\xi, \zeta \in \mathscr{A}$, and so is dense, since $\mathscr{A}^{2}$ is assumed dense. But a more compelling reason for making the assumption that $\mathscr{K} \cap i \mathscr{K}=\{0\}$ (besides the development in $\S \S 2$ and 3 ) is that it is easily seen that this condition is implied by the requirement that the map $\xi \rightarrow \xi^{\sharp}$, and so its restriction to $\mathscr{A}^{2}$, has a closed extension, which is one of the usual 
axioms for a Hilbert algebra. (The converse is true, but will only become clear in the course of the proof below - see also the remarks just before Lemma 2.3 of [18]). Thus we are ready to give a definition of a left Hilbert algebra which does not involve unbounded operators.

Definition 5.5. Let $\mathscr{A}$ be an involutive algebra with scalar product satisfying the axioms of Notation 5.1. Let $\mathscr{K}$ be the real subspace of $\mathscr{H}$ spanned by the $\xi^{\sharp} \xi$ for $\xi \in \mathscr{A}$. We will say that $\mathscr{A}$ is a left Hilbert algebra if $\mathscr{K} \cap i \mathscr{K}=\{0\}$.

We can now define the operators $J$ and $\Delta^{i t}$ on $\mathscr{H}$ using $\mathscr{C}$ as in $\S 3$. Our aim is then to prove the analogue of Theorem 4.2. To get started on this we need an analogue of Lemma 4.3. But in the setting of left Hilbert algebras we have no analogue of Sakai's Radon-Nikodym theorem or its variant, and so we will have to give a very different proof. We remark that in the lemma below we have interchanged the roles of $M$ and $M^{\prime}$ used in $\S 4$, because to avoid this we would have had to use right Hilbert algebras, which is not the usual convention. In the process we have interchanged $\lambda$ and $\bar{\lambda}$, and this will have the result that our later formulas are essentially the same as those in $\S 4$.

Lemma 5.6. Let $\xi \in \mathscr{K}$. Then for any $\lambda \in C$ with $\operatorname{Re}(\lambda)=1$ there is a unique $\eta \in i \mathscr{K}^{\perp}$ such that

$$
\langle\xi, \zeta\rangle=\operatorname{Re}(\lambda\langle\eta, \zeta\rangle) \text { for all } \zeta \in i \mathscr{K}^{\perp} .
$$

Moreover if $\xi$ is also in $\mathscr{A}$, then $\eta \in \mathscr{A}^{\prime}$ and $\eta^{\dagger}=\eta$.

Proof. Define a bilinear form, $B$, on $i \mathscr{K}^{\perp}$ by

$$
B(\eta, \zeta)=\operatorname{Re}(\lambda\langle\eta, \zeta\rangle) \text { for } \eta, \zeta \in i \mathscr{K}^{\perp} \text {. }
$$

Then $B(\eta, \eta)=\operatorname{Re}(\lambda\langle\eta, \eta\rangle)=\langle\eta, \eta\rangle$, so that $B$ is a scalar product inducing the original norm on $i \mathscr{K}^{\perp}$. Thus $i \mathscr{K}^{\perp}$ is a Hilbert space for the scalar product $B$.

Now for any $\xi \in \mathscr{K}$ the map $\zeta \mapsto\langle\xi, \zeta\rangle$ for $\zeta \in i \mathscr{K}^{\perp}$ is a real continuous functional on $i \mathscr{K}^{\perp}$, and so there exists $\eta \in i \mathscr{K}^{\perp}$ such that

$$
\langle\xi, \zeta\rangle=B(\eta, \zeta)=\operatorname{Re}(\lambda\langle\eta, \zeta\rangle) \text { for } \zeta \in i \mathscr{K}^{\perp} \text {. }
$$

This proves the first assertion.

The important part of the lemma is the second assertion, that if $\xi \in \mathscr{A}$ then $\eta \in \mathscr{A}^{\prime}$ and $\eta^{b}=\eta$. So suppose now that $\xi \in \mathscr{A}$. Let $a, b, c$ be the operators in $\mathscr{L}(\mathscr{A})^{\prime}$ associated to $\eta$ as in Lemma 5.4. 
What we will show is that now $\alpha$ is invertible, and that $\pi^{\prime}(\eta)=\alpha^{-1} b$. To show that $a$ is invertible we need to obtain estimates involving the spectral projections of $a$.

Let $e$ be any spectral projection of $\alpha$, and let

$$
\zeta=e b b^{*} \eta=e(1-a) a \eta .
$$

We show first that $\zeta \in \mathscr{A}^{\prime}$. Using Lemma 5.4 we find that for any $\xi_{1} \in \mathscr{A}$

$$
\begin{aligned}
\pi\left(\xi_{1}\right) \zeta & =\pi\left(\xi_{1}\right) e b b^{*} \eta=e b \pi\left(\xi_{1}\right) b^{*} \eta \\
& =e b c \xi_{1}=e(1-a) b \xi_{1},
\end{aligned}
$$

while

$$
\pi\left(\xi_{1}\right) b^{*} e b \eta=b^{*} e \pi\left(\xi_{1}\right) b \eta=b^{*} e(1-a) \xi_{1} .
$$

From the definition of $\mathscr{A}^{\prime}$ it follows that $\zeta \in \mathscr{A}^{\prime}$ and $\zeta^{b}=b^{*} e b \eta$.

Now equation (1) can be rewritten as

$$
2\langle\xi, \zeta\rangle=\lambda\langle\eta, \zeta\rangle+\bar{\lambda}\langle\zeta, \eta\rangle \text { for } \zeta \in i \mathscr{K}^{\perp} .
$$

But we have seen that if $\zeta \in \mathscr{A}^{\prime}$ and $\zeta^{b}=\zeta$ then $\zeta \in i \mathscr{K}^{\perp}$, so the above equation holds for such $\zeta$. By splitting elements of $\mathscr{A}^{\prime}$ into their real and imaginary parts it follows that

$$
2\left\langle\xi, \zeta^{b}\right\rangle=\lambda\left\langle\eta, \zeta^{b}\right\rangle+\bar{\lambda}\langle\zeta, \eta\rangle \text { for } \zeta \in \mathscr{A}^{\prime} .
$$

If we substitute the $\zeta$ defined above into this equation and take real parts, we obtain, since $\operatorname{Re} \lambda=1$ and $b b^{*}=a(1-a)$.

$$
\begin{aligned}
& \left\langle\eta, b^{*} e b \eta\right\rangle+\left\langle e b b^{*} \eta, \eta\right\rangle=2 \operatorname{Re}\left\langle\xi, b^{*} e b \eta\right\rangle \\
& \quad=2 \operatorname{Re}\langle e b \xi, e b \eta\rangle \leqq\|e b \xi\|^{2}+\|e b \eta\|^{2} \\
& =\langle e b \xi, b \xi\rangle+\left\langle\eta, b^{*} e b \eta\right\rangle .
\end{aligned}
$$

Using the inequality which results from this by cancelling identical terms, we obtain

$$
\begin{aligned}
& \langle e(1-a) a \eta, \eta\rangle=\left\langle e b b^{*} \eta, \eta\right\rangle \leqq\langle e b \xi, b \xi\rangle \\
& =\langle\pi(\xi) e a \eta, \pi(\xi) a \eta\rangle \leqq\|\pi(\xi)\|^{2}\left\langle e a^{2} \eta, \eta\right\rangle
\end{aligned}
$$

Now let $\varepsilon>0$ be given and let $e$ be the spectral projection for $\alpha$ on the interval $[0, \varepsilon]$. Since $\varepsilon e \geqq a e$, we obtain from the above inequality

$$
\begin{aligned}
& (1-\varepsilon)\langle e a \eta, \eta\rangle \leqq\langle e(1-a) a \eta, \eta\rangle \\
& \quad \leqq\|\pi(\xi)\|^{2}\left\langle e a^{2} \eta, \eta\right\rangle \leqq\|\pi(\xi)\|^{2} \varepsilon\langle e a \eta, \eta\rangle
\end{aligned}
$$

It follows that for small enough $\varepsilon$ (specifically for $\left.\varepsilon<\left(1+\|\pi(\xi)\|^{2}\right)^{-1}\right)$ we must have $e a \eta=0$. Then for any $\xi_{1} \in \mathscr{A}$ we have 


$$
e b \xi_{1}=e \pi\left(\xi_{1}\right) a \eta=\pi\left(\xi_{1}\right) e a \eta=0 .
$$

Thus $e b=0$, so that $e b b^{*}=e a(\dot{1}-a)=0$, and so $e a=0$, since $1-a$ has a bounded inverse on $e \mathscr{H}$. It will follow that $a$ itself has a bounded inverse once we show that zero is not an eigenvalue of $a$. But suppose that $a \xi_{1}=0$ for some $\xi_{1} \in \mathscr{H}$. Then $b^{*} \xi_{1}=0$ since $b b^{*}=a(1-a)$, and so $E\left(\xi_{1}, 0\right)=0$, that is, $\left(\xi_{1}, 0\right) \in \mathscr{N}^{\perp}$, where $E$ and $\mathscr{N}$ are defined as in Lemma 5.4. It follows that $\left\langle\xi_{1}, \zeta\right\rangle=0$ for all $\zeta \in \mathscr{A}$, so that $\xi_{1}=0$. Thus $a^{-1}$ is bounded.

Then for every $\xi_{1} \in \mathscr{A}$ we have

$$
\begin{aligned}
\pi\left(\xi_{1}\right) \eta & =a^{-1} \pi\left(\xi_{1}\right) a \eta=a^{-1} b \xi_{1}, \\
\left\langle\xi_{1}, a^{-1} b \xi_{1}\right\rangle & =\left\langle\xi_{1}, \pi\left(\xi_{1}\right) \eta\right\rangle=\left\langle\xi_{1}^{\#} \xi_{1}, \eta\right\rangle .
\end{aligned}
$$

Since $\eta \in i \mathscr{K}^{\perp}$, this last number is always real, and so $a^{-1} b$ is selfadjoint. Then from the definition of $\mathscr{A}^{\prime}$ it follows that $\eta \in \mathscr{A}^{\prime}$, $\pi^{\prime}(\eta)=a^{-1} b$, and $\eta^{b}=\eta$.

We are now in a position to imitate in the present setting the steps in $\S 4$ beginning at Corollary 4.4. We will let $R, T, J$ and $\Delta^{i t}$ be defined in terms of the $\mathscr{K}$ defined above, which we have seen satisfies the required nondegeneracy conditions.

Corollary 5.7. If $\xi \in \mathscr{A}^{2}$ then $T J \xi \in \mathscr{A}^{\prime}$ and $(T J \xi)^{b}=T J \xi^{\sharp}$.

Proof. Suppose first that $\xi \in \mathscr{A}^{2} \cap \mathscr{K}$. If we apply Lemma 5.6 with $\lambda=1$, we obtain an $\eta \in i \mathscr{C}^{\perp} \cap \mathscr{A}^{\prime}$ such that

$$
\langle\xi, \zeta\rangle=\operatorname{Re}\langle\eta, \zeta\rangle \cdot \text { for all } \zeta \in i \mathscr{K}^{\perp} \text {. }
$$

This means that $\eta$ is the orthogonal projection of $\xi$ onto $i \mathscr{K}^{\perp}$, so $\eta=(1-Q) \xi$. Since $P \xi=\xi$, we have

$$
T J \xi=(P-Q) \xi=(1-Q) \xi=\eta \text {. }
$$

The general result now follows by polarization and conjugate linearity.

Lemma 5.8. Let $\xi \in \mathscr{A} \cap \mathscr{K}$. Then for any $\lambda \in C$ with $\operatorname{Re}(\lambda)>0$ there is an $\eta \in \mathscr{A}^{\prime}$ such that $\eta^{\mathrm{b}}=\eta$ and

$$
T J \pi(\xi) J T=\lambda(2-R) \pi^{\prime}(\eta) R+\bar{\lambda} R \pi^{\prime}(\eta)(2-R) .
$$

Furthermore $J T \xi=(\lambda(2-R)+\bar{\lambda} R) \eta$.

Proof. We may restrict ourselves to the case $\operatorname{Re}(\lambda)=1$. Then by Lemma 5.6 (with $\eta$ replaced by $\eta / 2$ ), there is an $\eta \in \mathscr{A}^{\prime} \cap i \mathscr{K}^{\perp}$ such that 


$$
\langle\xi, \zeta\rangle=\lambda\langle\eta, \zeta\rangle+\bar{\lambda}\langle\zeta, \eta\rangle \text { for all } \zeta \in i \mathscr{K}^{\perp} \text {. }
$$

Since $\mathscr{H}^{\prime} \cong i \mathscr{K}^{\perp}+\mathscr{K}^{\perp}$, this relation will hold for arbitrary if we change the third $\zeta$ to $\zeta^{\circ}$. If we then substitute $\zeta_{2}^{\circ} \circ \zeta_{1}$ for $\zeta$, where $\zeta_{2}, \zeta_{1} \in \mathscr{A}^{\prime}$, we obtain

$$
\left\langle\pi(\xi) \zeta_{2}, \zeta_{1}\right\rangle=\lambda\left\langle\zeta_{2} \circ \eta, \zeta_{1}\right)+\bar{\lambda}\left\langle\zeta_{2}, \zeta_{1} \circ \eta\right\rangle .
$$

Now let $\xi_{1}, \xi_{2} \in \mathscr{A}^{2}$, and let $\zeta_{i}=T J \xi_{2}$ for $i=1,2$. Then $\zeta_{2} \in \mathscr{A}^{\prime}$ and $\zeta_{i}^{b}=T J \xi_{i}^{\sharp}$ by Corollary 5.7. Substituting these in the above equation and rearranging, using Proposition 3.1, we obtain

$$
\left\langle\xi_{1}, T J \pi(\xi) T J \xi_{2}\right\rangle=\lambda\left\langle\xi_{1}, T J\left(\zeta_{2} \circ \eta\right)\right\rangle+\bar{\lambda}\left\langle T J\left(\zeta_{1} \circ \eta\right), \xi_{2}\right\rangle .
$$

Now for any $\zeta \in \mathscr{A}^{\prime}$ with $\zeta^{b}=\zeta$ we have $\zeta \in i \mathscr{K}^{\perp}$ so that $Q \zeta=0$ and thus

$$
T J \zeta=(P-Q) \zeta=(P+Q) \zeta=R \zeta .
$$

Similarly, if $\xi_{3} \in \mathscr{A} \cap \mathscr{\mathscr { C }}$ then $P \xi_{3}=\xi_{3}$ so that

$$
T J \xi_{3}=(P-Q) \xi_{3}=(2-P-Q) \xi_{3}=(2-R) \xi_{3} .
$$

Using the fact that $R$ is linear while $T J$ is conjugate linear, we see that for any $\zeta \in \mathscr{A}^{\prime}$ and any $\xi_{3} \in \mathscr{A}^{2}$ we have

$$
T J \zeta=R \zeta^{b}, \quad T J \xi_{3}=(2-R) \xi_{3}^{\sharp} .
$$

It follows from this, Corollary 5.7 and $\eta^{\beta}=\eta$, that

$$
T J\left(\zeta_{i} \circ \eta\right)=R\left(\eta^{b} \circ \zeta_{i}^{0}\right)=R \pi^{\prime}(\eta) T J \xi_{i}^{\ddagger}=R \pi^{\prime}(\eta)(2-R) \xi_{i} .
$$

Substituting these expressions in (2) we obtain

$$
\left\langle\xi_{1}, T J \pi(\xi) J T \xi_{2}\right\rangle=\lambda\left\langle\xi_{1}, R \pi^{\prime}(\eta)(2-R) \xi_{2}\right\rangle+\bar{\lambda}\left\langle R \pi^{\prime}(\eta)(2-R) \xi_{1}, \xi_{2}\right\rangle .
$$

Since $\xi_{1}$ and $\xi_{2}$ are arbitrary elements of $\mathscr{A}^{2}$, and $\mathscr{A}^{2}$ is dense in $\mathscr{H}$, we obtain the first equation of the Lemma.

To obtain the second equation let $\xi_{1} \in \mathscr{A} \cap \mathscr{K}$, let $\zeta_{1}=T J \xi_{1}$ as above, and substitute this in (1). We obtain, after rearranging,

$$
\left\langle\xi_{1}, T J \xi\right\rangle=\lambda\left\langle\xi_{1}, T J \eta\right\rangle+\bar{\lambda}\left\langle T J \xi_{1}, \eta\right\rangle \text {. }
$$

But, as above, $T J \xi_{1}=(2-R) \xi_{1}$, and $T J \eta=R \eta$, since $\eta \in i \mathscr{C}^{\perp}$. Thus

$$
\left\langle\xi_{1}, T J \xi\right\rangle=\lambda\left\langle\xi_{1}, R \eta\right\rangle+\bar{\lambda}\left\langle\xi_{1},(2-R) \eta\right\rangle,
$$

from which the desired equation follows.

We remark that the first equation of the above lemma is the analogue of Lemma 4.5 , and we could continue as in $\S 4$ to show that $J \Delta^{i t} \mathscr{L}(\mathscr{A}) \Delta^{-i t} J \subseteq \mathscr{L}(\mathscr{A})^{\prime}$. But we need to prove more, namely 
that if $\xi \in \mathscr{A}$ then $J \Delta^{i t} \xi \in \mathscr{A}^{\prime}$ and

$$
\pi^{\prime}\left(J \Delta^{i t} \xi\right)=J \Delta^{i t} \pi(\xi) \Delta^{-i t} J,
$$

and it is for this that we need the second equation of the above lemma, as well as of the next lemma (the analogue of Lemma 4.7).

LEMMA 5.9. Let $\lambda=e^{i \phi / 2}$ where $-\pi<\phi<\pi$, and let $\xi$ and $\eta$ be as in Lemma 5.8. Then

$$
\begin{aligned}
\pi^{\prime}(\eta) & =\int_{-\infty}^{+\infty} e^{-\phi t}\left(e^{\pi t}+e^{-\pi t}\right)^{-1} \Delta^{i t} J \pi(\xi) J \Delta^{-i t} d t, \\
\eta & =\int_{-\infty}^{\infty} e^{-\phi t}\left(e^{\pi t}+e^{-\pi t}\right)^{-1} \Delta^{i t} J \xi d t .
\end{aligned}
$$

Proof. The first formula follows from Lemma 5.8 and Lemma 4.6 just as Lemma 4.7 does from Lemma 4.5. To prove the second formula, apply Lemma 4.6 to the function

$$
f(z)=\left\langle R^{-z+1 / 2}(2-R)^{z+1 / 2} \eta, \xi_{1}\right\rangle
$$

for any $\xi_{1} \in \mathscr{H}$, and argue as in the proof of Lemma 4.7, using the second equation of Lemma 5.8.

Lemma 5.10. Let $\xi_{0} \in \mathscr{A}$ and let $\xi=\xi_{0}^{\sharp} \xi_{0}$. Then $\Delta^{i t} J \xi \in \mathscr{A}^{\prime}$ for all real $t$, and

$$
\pi^{\prime}\left(\Delta^{i t} J \xi\right)=\Delta^{i t} J \pi(\xi) J \Delta^{-i t}, \quad\left(\Delta^{i t} J \xi\right)^{b}=\Delta^{i t} J \xi .
$$

Proof. Let $\lambda=e^{i \phi / 2}$ where $-\pi<\phi<\pi$, and let $\eta$ be defined as in Lemma 5.8 in terms of $\xi$ and $\lambda$. Now $0=\pi(\zeta) \eta-\pi^{\prime}(\eta) \zeta$ for any $\zeta \in \mathscr{A}$, and so from Lemma 5.9 we obtain

$$
0=\int_{-\infty}^{\infty} e^{-\phi t}\left(e^{\pi t}+e^{-\pi t}\right)^{-1}\left(\pi(\zeta) J \Delta^{i t} \xi-\Delta^{i t} J \pi(\xi) J \Delta^{-i t} \zeta\right) d t
$$

for all $\phi$ with $-\pi<\phi<\pi$. Arguing as in the proof of Lemma 4.8 we conclude that

$$
\pi(\zeta) J \Delta^{i t} \xi=\Delta^{i t} J \pi(\xi) J \Delta^{-i t} \zeta \text { for all real } t \text {. }
$$

Now $\xi=\xi^{\sharp}$, and so $\pi(\xi)$ is self-adjoint, as is $\Delta^{-i t} J \pi(\xi) J \Delta^{i t}$. Then it follows from the definition of $\mathscr{A}^{\prime}$ that $\Delta^{i t} J \xi \in \mathscr{A}^{\prime}$, that $\left(\Delta^{i t} J \xi\right)^{b}=$ $\Delta^{i t} J \xi$, and that

$$
\pi^{\prime}\left(\Delta^{i t} J \xi\right) \zeta=\Delta^{i t} J \pi(\xi) J \Delta^{-i t} \zeta \text { for } \zeta \in \mathscr{H} .
$$

The argument from now on differs a little from that in [18] in that we will focus earlier on showing that the role of $\mathscr{A}^{\prime}$ is almost 
symmetric to that of $\mathscr{A}\left(\mathscr{A}^{\prime}\right.$ will be full while $\mathscr{A}$ need not be).

LEMMA 5.11. The *-algebra $\mathscr{A}^{\prime}$ equipped with the scalar product from $\mathscr{H}$ is a left Hilbert algebra whose left von Neumann algebra is $\mathscr{L}(\mathscr{A})^{\prime}$

Proof. It is clear that $\mathscr{A}^{\prime}$ satisfies properties 1 and 2 of Definition 5.1. Furthermore, setting $t=0$ in Lemma 5.10 we find that $J \mathscr{A}^{2} \cong \mathscr{A}^{\prime}$. Since $\mathscr{A}^{2}$ is dense in $\mathscr{H}$, it follows that $\mathscr{A}^{\prime}$ is also. But to show that property 3 holds, we need to show that the identity operator on $\mathscr{H}$ is in the strong operator closure of $\pi^{\prime}\left(\mathscr{A}^{\prime}\right)$. Now since the representation $\pi$ of $\mathscr{A}$ on $\mathscr{H}$ is non-degenerate, we can find a net $\xi_{i}$ of elements of $\mathscr{A}$ such that $\pi\left(\xi_{i}\right)$ converges strongly to the identity operator and $\left\|\pi\left(\xi_{i}\right)\right\| \leqq 1$. The same will then be true of $\pi\left(\xi_{i}^{\sharp} \xi_{i}\right)$. But if we let $\zeta_{i}=J\left(\xi_{i}^{\#} \xi_{i}\right)$, then, setting $t=0$ in Lemma 5.10 we see that $\zeta_{i} \in \mathscr{A}^{\prime}$ and that $\pi^{\prime}\left(\zeta_{i}\right)=J \pi\left(\xi_{i}^{\sharp} \xi_{i}\right) J$, which also converges to the identity. Thus $\left(\mathscr{A}^{\prime}\right)^{2}=\pi\left(\mathscr{A}^{\prime}\right) \mathscr{A}^{\prime}$ is dense in $\mathscr{H}$, so that property 3 holds.

To show that $\mathscr{X}^{\prime}$ is a left Hilbert algebra, we must show that if $\mathscr{K}^{\prime \prime}$ denotes the (closed) real subspace of $\mathscr{H}$ spanned by the $\eta^{\text {b}} \circ \eta$ for $\eta \in \mathscr{A}^{\prime}$, then $\mathscr{K}^{\prime} \cap i \mathscr{K}^{\prime}=\{0\}$. But if $\zeta=\eta^{b} \circ \eta$ then $\zeta^{b}=\zeta$ so that, as seen earlier, $\zeta \in i \mathscr{K}^{\perp}$. That is, $\mathscr{K}^{\prime} \cong i \mathscr{K}^{\perp}$. But $\mathscr{K}+i \mathscr{K}$ is dense, and so $\mathscr{K}^{\perp} \cap i \mathscr{K}^{\perp}=\{0\}$. Thus also $\mathscr{K}^{\prime} \cap i \mathscr{K}^{\prime}=\{0\}$.

Finally, we show that $\pi^{\prime}\left(\mathscr{A}^{\prime}\right)$ is strongly dense in $\mathscr{L}(\mathscr{A})^{\prime}$. Let $x^{\prime} \in \mathscr{L}\left(\mathscr{A}^{\prime}\right)$ and let $\zeta, \eta \in \mathscr{A}^{\prime}$. Then for $\xi \in \mathscr{A}$ we have

$$
\begin{gathered}
\pi^{\prime}(\zeta) x^{\prime} \pi^{\prime}(\eta) \xi=\pi^{\prime}(\zeta) x^{\prime} \pi(\xi) \eta=\pi(\xi) \pi^{\prime}(\zeta) x^{\prime} \eta \\
\left(\pi^{\prime}(\zeta) x^{\prime} \pi^{\prime}(\eta)\right)^{*} \xi=\pi^{\prime}(\eta)^{*} x^{\prime *} \pi(\xi) \zeta^{b}=\pi(\xi) \pi^{\prime}(\eta)^{*} x^{\prime *} \zeta^{b} .
\end{gathered}
$$

It follows from the definition of $\mathscr{A}^{\prime}$ that $\pi^{\prime}(\zeta) x^{\prime} \eta \in \mathscr{A}^{\prime}$ and

$$
\pi^{\prime}\left(\pi^{\prime}(\zeta) x^{\prime} \eta\right)=\pi^{\prime}(\zeta) x^{\prime} \pi^{\prime}(\eta) .
$$

Thus $\pi^{\prime}(\zeta) x^{\prime} \pi^{\prime}(\eta) \in \pi\left(\mathscr{A}^{\prime}\right)$. If we now let $\zeta$ and $\eta$ run through the net $\zeta_{i}$ of the previous paragraph, we see that $\pi^{\prime}\left(\zeta_{i}\right) x^{\prime} \pi^{\prime}\left(\zeta_{i}\right)$ converges strongly to $x^{\prime}$. Thus $\pi^{\prime}\left(\mathscr{A}^{\prime}\right)$ is strongly dense in $\mathscr{L}(\mathscr{A})^{\prime}$.

Actually, we can now prove that $\mathscr{K}^{\prime}=i \mathscr{K}^{\perp}$, so that the $J$ corresponding to $\mathscr{A}^{\prime}$ is the same as that for $\mathscr{A}$, while the $\Delta^{i t}$ corresponding to $\mathscr{A}^{\prime}$ is the $\mathscr{A}^{-i t}$ for $\mathscr{A}$. The proof of the first part of the next lemma is inspired by the proof of Lemma 3.4 of [13].

LEMMA 5.12. $\mathscr{K}$ is the closure of the set of self-adjoint elements of $\mathscr{A}$, and $\mathscr{K}^{\prime}=i \mathscr{K}^{\perp}$. 
Proof. Of course $\mathscr{K}$ is contained in the closure of the set of self-adjoint elements of $\mathscr{A}$. Suppose, conversely, that $\xi \in \mathscr{A}$ with $\xi^{\#}=\xi$. Now if $p$ is any real polynomial with no constant term, then $p(\pi(\xi)) \xi \in \mathscr{r}$, as is seen by real polarization. But we can approximate the range projection, $e$, of $\pi(\xi)$ in the strong operator topology by such $p(\pi(\xi))$, and so $e \xi \in \mathscr{K}$. However, for any $\eta \in \mathscr{A}^{\prime}$

$$
\pi^{\prime}(\eta) e \xi=e \pi^{\prime}(\eta) \xi=e \pi(\xi) \eta=\pi(\xi) \eta=\pi^{\prime}(\eta) \xi,
$$

and so, since we now know that $\pi^{\prime}$ is nondegenerate, $e \xi=\xi$. Thus $\xi \in \mathscr{K}$.

To show that $\mathscr{K}^{\prime}=i \mathscr{K}^{\perp}$, note first that since we know that $\mathscr{A}^{\prime}$ is a left Hilbert algebra, we can apply to $\mathscr{A}^{\prime}$ the result just proved to conclude that $\mathscr{K}^{\prime}$ is the closure of the set of self-adjoint elements of $\mathscr{A}^{\prime}$. Thus it suffices to show that the set of selfadjoint elements of $\mathscr{A}^{\prime}$ is dense in $i \mathscr{K}^{\perp}$. That this set is contained in $i \mathscr{K}^{\perp}$ was discussed just after Proposition 5.3. That this set is dense follows immediately from Proposition 5.10 (with $t=0$ ) and the remark just after Proposition 2.2.

Since it follows that the $J$ for $\mathscr{A}^{\prime}$ is the same as that for $\mathscr{A}$, while the $\Delta^{i t}$ for $\mathscr{A}^{\prime}$ is the $\Delta^{-i t}$ for $\mathscr{A}$, we can now easily obtain the main results.

THEOREM 5.13. Let $\mathscr{A}$ be a left Hilbert algebra. Then

$$
J \mathscr{L}(\mathscr{A}) J=\mathscr{L}(\mathscr{A})^{\prime}, \quad \Delta^{i t} \mathscr{L}(\mathscr{A}) \Delta^{-i t}=\mathscr{L}(\mathscr{A}) \text { for all real } t \text {. }
$$

Proof. From Lemma 5.10 we obtain

$$
\Delta^{i t} J \mathscr{L}(\mathscr{A}) J \Delta^{-i t} \cong \mathscr{L}(\mathscr{A})^{\prime} \text {. }
$$

Then by symmetry

$$
\Delta^{i t} J \mathscr{L}\left(\mathscr{A}^{\prime}\right) J \Delta^{-i t} \cong \mathscr{L}\left(\mathscr{A}^{\prime}\right)^{\prime} .
$$

But $\left.\mathscr{L}(\mathscr{A})^{\prime}\right)=\mathscr{L}(\mathscr{A})^{\prime}$ by Lemma 5.11. From this the desired results follow.

The following is a sharpening of Lemma 5.10.

THEOREM 5.14. Let $\mathscr{A}$ be a left Hilbert algebra. If $\xi \in \mathscr{A}$, then $\Delta^{i t} J \xi \in \mathscr{L}^{\prime}$ for all real $t$, and

$$
\pi^{\prime}\left(\Delta^{i t} J \xi\right)=\Delta^{i t} J \pi(\xi) J \Delta^{-i t}, \quad\left(\Delta^{i t} J \xi\right)^{b}=\Delta^{i t} J \xi^{\#} .
$$

Proof. It suffices to prove the theorem when $\xi=\xi^{\sharp}$. Now if 
$p$ is any real polynomial with no constant term, then it follows from Lemma 5.10, as in the proof of Lemma 5.12, that $\Delta^{i t} J p(\pi(\xi)) \xi \in \mathscr{A}^{\prime}$, and that for any $\eta \in \mathscr{A}$ we have

$$
\pi(\eta) \Delta^{i t} J p(\pi(\xi)) \xi=\Delta^{i t} J p(\pi(\xi)) \pi(\xi) J \Delta^{-i t} \eta \text {. }
$$

If we then let the $p(\pi(\xi))$ approximate the range projection, $e$, of $\pi(\xi)$, so that $e \xi=\xi$ as in the proof of Lemma 5.12, we obtain

$$
\pi(\eta) \Delta^{i t} J \xi=\Delta^{i t} J \pi(\xi) J \Delta^{-i t} \eta \text {. }
$$

The desired result now follows from the definition of $\mathscr{A}^{\prime}$.

From Theorem 5.14 applied to $\mathscr{A}$ as well as $\mathscr{A}^{\prime}$ we can easily obtain the results about achieved (i.e. full) left Hilbert algebras contained in [18]. We include them here for the readers' convenience.

Since $\mathscr{A}^{\prime}$ is a left Hilbert algebra, we can define $\mathscr{A}^{\prime \prime}=\left(\mathscr{A}^{\prime}\right)^{\prime}$. If $\xi \in \mathscr{A}$, then for any $\eta \in \mathscr{A}^{\prime}$ we have

$$
\pi^{\prime}(\eta) \xi=\pi(\xi) \eta, \quad \pi^{\prime}(\eta) \xi^{\sharp}=\pi(\xi)^{*} \eta,
$$

so that $\mathscr{A} \cong \mathscr{A}^{\prime \prime}$, and it is consistent to use the notation $\pi$ and \# for the representation and involution of $\mathscr{A}^{\prime \prime}$. Of course the left von Neumann algebra of $\mathscr{A}^{\prime \prime}$ is again $\mathscr{L}\left(\mathscr{A}^{\prime}\right)^{\prime}=\mathscr{L}(\mathscr{A})$.

One can also form $\mathscr{A}^{\prime \prime \prime}=\left(\mathscr{A}^{\prime \prime}\right)^{\prime}$. But if $\eta \in \mathscr{A}^{\prime \prime \prime}$, then for all $\xi \in \mathscr{A}^{\prime \prime} \cdot$ we have

$$
\pi(\xi) \eta=\pi^{\prime}(\eta) \xi, \quad \pi(\xi) \eta^{b}=\pi^{\prime}(\eta)^{*} \xi .
$$

Since $\mathscr{A} \subseteq \mathscr{A}^{\prime \prime}$, this also holds for $\xi \in \mathscr{A}$, and thus $\eta \in \mathscr{A}^{\prime}$. Hence $\mathscr{A}^{\prime \prime \prime}=\mathscr{A}^{\prime}$. Similarly $\mathscr{A}^{\prime \prime \prime \prime}=\mathscr{A}^{\prime \prime}$, etc.

A left Hilbert algebra is said to be achieved (or full) if $\mathscr{A}=$ $\mathscr{A}^{\prime \prime}$. The remarks just above show that $\mathscr{A}^{\prime}$ is always achieved. Also, $\mathscr{A}^{\prime \prime}$ is achieved, so that any left Hilbert algebra is contained in a left Hilbert algebra $\mathscr{A}^{\prime \prime}$ which is achieved and is such that $\mathscr{L}(\mathscr{A})=\mathscr{L}\left(\mathscr{A}^{\prime \prime}\right)$. Applying Theorem 5.14 successively to $\mathscr{A}$ and $\mathscr{A}^{\prime}$ we obtain

THEOREm 5.15. If $\mathscr{A}$ is an achieved left Hilbert algebra, then $J \mathscr{A}=\mathscr{A}^{\prime}$, and

$$
\pi^{\prime}(J \xi)=J \pi(\xi) J \text { for all } \xi \in \mathscr{A} .
$$

Furthermore, $\Delta^{i t} \mathscr{A}=\mathscr{A}$, and for $\xi \in \mathscr{A}$

$$
\pi\left(\Delta^{i t} \xi\right)=\Delta^{i t} \pi(\xi) \Delta^{-i t} \text { for all real } t .
$$

6. Appendix. In this appendix we investigate the relation be- 
tween our operators $R, T, J$ and the traditional operators of the theory, namely $S, F$ and $\Delta . \quad$ As in $\S 2$ let $\mathscr{K}$ and $\mathscr{L}$ be the (closed) subspaces of a real Hilbert space $\mathscr{H}$ satisfying the nondegeneracy condition that $\mathscr{K} \cap \mathscr{L}=\{0\}$, and $\mathscr{K}+\mathscr{L}$ is dense. Let $P$ and $Q$ denote the orthogonal projections on $\mathscr{K}$ and $\mathscr{L}$ respectively, and let $R=P+Q$, TJ $=P-Q$.

Now we can also associate to the pair ( $\mathscr{K}, \mathscr{L}$ ) certain (usually) unbounded operators. We already mentioned in $\S 2$ the operator $S$, whose domain, $D(S)$, is $\mathscr{K}+\mathscr{L}$ and which is defined by

$$
S(\xi+\eta)=\xi-\eta \text { for } \xi \in \mathscr{K}, \eta \in \mathscr{L} \text {. }
$$

Then $S$ is a closed, densely defined, operator. Now since the pair $\left(\mathscr{L}^{\perp}, \mathscr{K}^{\perp}\right)$ also satisfies the nondegeneracy condition, we can define an operator $F$ whose domain, $D(F)$, is $\mathscr{L}^{\perp}+\mathscr{K}^{\perp}$, and which is defined by

$$
F\left(\xi_{1}+\eta_{1}\right)=\xi_{1}-\eta_{1} \text { for } \xi_{1} \in \mathscr{L}^{\perp}, \eta_{1} \in \mathscr{K}^{\perp} .
$$

Again $F$ is closed and densely defined. The operators $S$ and $F$ correspond to the traditional ones of Tomita-Takesaki theory. Their relations with $R, T$ and $J$ are given by:

Proposition.

(1) $F=J S J, F=S^{*}$.

(2) If $\xi \in D(S)$, then $(2-R) S \xi=J T \xi$,

If $\eta \in D(F)$, then $R F \eta=J T \eta$.

(3) If $\Delta=(2-R) R^{-1}$, then we have the polar decompositions

$$
S=J \Delta^{1 / 2}, \quad F=J \Delta^{-1 / 2} .
$$

Proof. According to Lemma $2.3 \mathrm{~J}$ is an orthogonal self-adjoint operator which maps the pair $(\mathscr{K}, \mathscr{L})$ to the pair $\left(\mathscr{L}^{\perp}, \mathscr{K}^{\perp}\right)$, and so it is apparent that $F=J S J$. For $\xi \in \mathscr{K}, \eta \in \mathscr{L}, \xi_{1} \in \mathscr{L}^{\perp}, \eta_{1} \in \mathscr{K}^{\perp}$ we have

$$
\begin{gathered}
\left\langle S(\xi+\eta), \xi_{1}+\eta_{1}\right\rangle=\left\langle\xi-\eta, \xi_{1}+\eta_{1}\right\rangle=\left\langle\xi, \xi_{1}\right\rangle-\left\langle\eta, \eta_{1}\right\rangle \\
=\left\langle\xi+\eta, \xi_{1}-\eta_{1}\right\rangle=\left\langle\xi+\eta, F\left(\xi_{1}+\eta_{1}\right)\right\rangle .
\end{gathered}
$$

Thus $F \cong S^{*}$. On the other hand, if $\zeta \in D\left(S^{*}\right)$, then

$$
\langle\xi-\eta, \zeta\rangle=\langle S(\xi+\eta), \zeta\rangle=\left\langle\xi+\eta, S^{*} \zeta\right\rangle
$$

for all $\xi \in \mathscr{K}$ and $\eta \in \mathscr{L}$. If $\eta=0$ this implies that $\zeta-S^{*} \zeta \in \mathscr{K}^{\perp}$, while if $\xi=0$ it implies that $\zeta+S^{*} \zeta \in \mathscr{L}^{\perp}$. Consequently $\zeta \in \mathscr{L}^{\perp}+$ $\mathscr{K}^{\perp}$. Thus $D\left(S^{*}\right)=\mathscr{L}^{\perp}+\mathscr{K}^{\perp}$, so that $F=S^{*}$. This proves part 1 . Let $\xi \in \mathscr{K}$ and $\eta \in \mathscr{L}$. Then 


$$
\begin{aligned}
& (2-R) S(\xi+\eta)=(2-P-Q)(\xi-\eta) \\
& \quad=(1-P)(\xi-\eta)+(1-Q)(\xi-\eta)=-(1-P) \eta+(1-Q) \xi \\
& \quad=-(Q-P) \eta+(P-Q) \xi=(P-Q)(\xi+\eta)=J T(\xi+\eta) .
\end{aligned}
$$

This proves the first equation of part 2. The second is proved in a similar way.

From part 1 we have

$$
(J S)^{*}=S^{*} J=F J=J S,
$$

so that $J S$ is self-adjoint. On the other hand, part 2 shows that if $\xi \in D(S)$ then $R J S \xi=T \xi$, or, since $T=(2-R)^{1 / 2} R^{1 / 2}$.

$$
R^{1 / 2} J S \xi=(2-R)^{1 / 2} \xi \text {. }
$$

If we set $\Delta=(2-R) R^{-1}$, this implies that $\xi \in D\left(\Delta^{1 / 2}\right)$, and that $J S \subseteq \Delta^{1 / 2}$. But by the maximality of self-adjoint operators, we must have equality. The second equation of part 3 can be proved in a similar way, or by using the relation $J \Delta^{1 / 2} J=\Delta^{-1 / 2}$.

\section{REFERENCES}

1. H. Araki, Some properties of modular conjugation operator of von Neumann algebras and a non-commutative Radon-Nikodym theorem with a chain rule, Pacific J. Math., 50 (1974), 309-354.

2. F. Combes, Poid associé à une algèbre Hilbertienne à gauche, Comp. Math., 23 (1971), 49-77.

3. A. Connes, Une classification des facteurs de type III. Ann. Scient Ec. Norm. Sup., 6 (1973), 133-252.

4. J. Dixmier, Positions relative de deux variétés linéaires fermées dans un espace de Hilbert, Rev. Sci., 86 (1948), 387-399.

5 . - Les algèbres d'opérateures dans l'espace hilbertien, $2^{\text {nd }}$ ed. Gauthièr-Villars, Paris, 1969

6. J.-P. Eckmann and K. Osterwalder, An application of Tomita's theory of modular Hilbert algebras: Duality for free Bose fields, J. Functional Analysis, 13 (1973), 1-12.

7. P. R. Halmos, Two subspaces, Trans. Amer. Math. Soc., 144 (1969), 381-389.

8. R. Herman, Perturbations of the modular automorphism group, Comm. Math. Phys., 28 (1972), 237-243.

9. M. A. Rieffel, A commutation theorem and duality for free Bose fields, Comm. Math. Phys., 39 (1974), 153-164.

10. M. A. Rieffel and A. Van Daele, The commutation theorem for tensor products of von Neumann algebras, Bull. London Math. Soc., 7 (1975), 257-260.

11. W. Rudin, Real and Complex Analysis, McGraw-Hill, New York, 1966.

12. S. Sakai, $C^{*}$-algebras and $W^{*}$-algebras, Springer-Verlag, Berlin-Heidelberg-New York, 1971.

13. M. Takesaki, Tomita's theory of modular Hilbert algebras and its applications, Springer Lecture Notes no. 128, Berlin-Heidelberg-New York, 1970.

14. - States and automorphisms of operator algebras. Standard representations and the Kubo-Martin-Schwinger Boundary Condition. In Statistical Mechanics and Mathematical Problems, Lecture Notes in Physics no. 20, Springer-Verlag, BerlinHeidelberg-New York, 1973. 
15. M. Takesaki, Duality for crossed products and the structure of von Neumann algebras of type III, Acta Math., 131 (1973), 249-310.

16. M. Tomita, Standard forms of von Neumann algebras, The $\mathrm{V}^{\text {th }}$ functional analysis symposium of the Mathematical Society of Japan, Sendai (1967).

17. - Quasi-standard von Neumann algebras, Mimeographed notes, 1967.

18. A. Van Daele, $A$ new approach to the Tomita-Takesaki theory of generalized Hilbert algebras, J. Functional Analysis, 15 (1974), 378-393.

19. - The Tomita-Takesaki theory for von Neumann algebras with a separating and cyclic vector, Proceedings of the Varenna conference (1973) on $C^{*}$-algebras and their applications to statistical mechanics and quantum field theory. To appear.

20. M. Winnink, Algebraic aspects of the Kubo-Martin-Schwinger condition, Cargèse Lectures in Physics, vol. 4, Gordon and Breach, New York-London-Paris, 1970.

21. S. L. Woronowicz, On the purification of factor states, Comm. Math. Phys., 28 (1972), 221-235.

22. L. Zsidó, A proof of Tomita's fundamental theorem in the theory of standard von Neumann algebras, preprint.

Received October 13, 1976.

University of CALIFornia, Berkeley 94720

AND

UNIVERSITY OF LEUVEN

Celestijnenlaan $200 \mathrm{~B}$

B-3030 HEVERLEE

BELGIUM 



\section{PACIFIC JOURNAL OF MATHEMATICS}

EDITORS

RICHARD ARENS (Managing Editor)

University of California

Los Angeles, California 90024

R. A. Beaumont

University of Washington

Seattle, Washington 98105
J. DUGUNDJI

Department of Mathematics

University of Southern Californı

Los Angeles, California 90007

D. Gilbarg and J. Milgram

Stanford University

Stanford, California 94305

\section{ASSOCIATE EDITORS}

E. F. BECKENBACH

B. H. NeumanN

F. WOLF

K. YoSHIDA

\section{SUPPORTING INSTITUTIONS}

UNIVERSITY OF BRITISH COLUMBIA

CALIFORNIA INSTITUTE OF TECHNOLOGY

UNIVERSITY OF CALIFORNIA

MONTANA STATE UNIVERSITY

UNIVERSITY OF NEVADA

NEW MEXICO STATE UNIVERSITY

OREGON STATE UNIVERSITY

UNIVERSITY OF OREGON

OSAKA UNIVERSITY
UNIVERSITY OF SOUTHERN CALIFORNIA

STANFORD UNIVERSITY

UNIVERSITY OF TOKYO

UNIVERSITY OF UTAH

WASHINGTON STATE UNIVERSITY

UNIVERSITY OF WASHINGTON

AMERICAN MATHEMATICAL SOCIETY

NAVAL WEAPONS CENTER 


\section{Pacific Journal of Mathematics}

Vol. 69 , No. 1

May, 1977

V. V. Anh and P. D. Tuan, On starlikeness and convexity of certain analytic

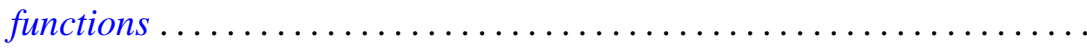

Willard Ellis Baxter and L. A. Casciotti, Rings with involution and the prime

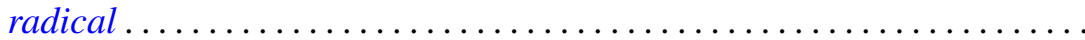

Manuel Phillip Berriozabal, Hon-Fei Lai and Dix Hayes Pettey,

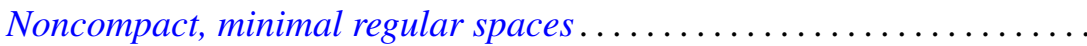

Sun Man Chang, Measures with continuous image law ................

John Benjamin Friedlander, Certain hypotheses concerning

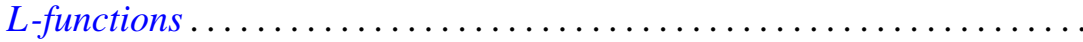

Moshe Goldberg and Ernst Gabor Straus, On characterizations and

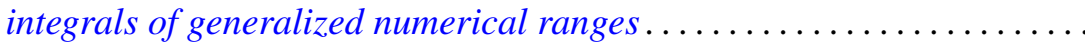

Pierre A. Grillet, On subdirectly irreducible commutative semigroups ...... 55

Robert E. Hartwig and Jiang Luh, On finite regular rings ..............

Roger Hugh Hunter, Fred Richman and Elbert A. Walker, Finite direct sums of cyclic valuated p-groups ........................... 97

Atsushi Inoue, On a class of unbounded operator algebras. III ......... 105

Wells Johnson and Kevin J. Mitchell, Symmetries for sums of the Legendre

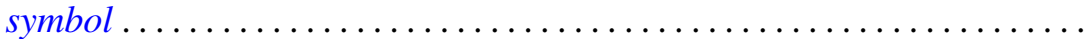

Jimmie Don Lawson, John Robie Liukkonen and Michael William Mislove,

Measure algebras of semilattices with finite breadth

Glenn Richard Luecke, A note on spectral continuity and on spectral properties of essentially $G_{1}$ operators ...............

Takahiko Nakazi, Invariant subspaces of weak-* Dirichlet algebras . .

James William Pendergrass, Calculations of the Schur group ...

Carl Pomerance, On composite $n$ for which $\varphi(n) \mid n-1$. II. . .

Marc Aristide Rieffel and Alfons Van Daele, A bounded operator approach to Tomita-Takesaki theory........................

Daniel Byron Shapiro, Spaces of similarities. IV. $(s, t)$-families ...

Leon M. Simon, Equations of mean curvature type in 2 independent variables.

Joseph Nicholas Simone, Metric components of continuous images of ordered compacta ............................

William Charles Waterhouse, Pairs of symmetric bilinear forms in

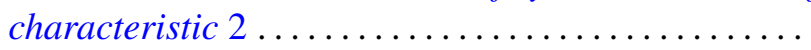

